\title{
Efficient and Robust Certification of Genuine Multipartite Entanglement in Noisy Quantum Error Correction Circuits
}

\author{
Andrea Rodriguez-Blanco $\odot,{ }^{1,}{ }^{*}$ Alejandro Bermudez, ${ }^{1}$ Markus Müller, ${ }^{2,3,4}$ and Farid Shahandeh ${ }^{2}$ \\ ${ }^{1}$ Departamento de Física Teorica, Universidad Complutense, Madrid 28040, Spain \\ ${ }^{2}$ Department of Physics, Swansea University, Singleton Park, Swansea SA2 8PP, United Kingdom \\ ${ }^{3}$ Institute for Quantum Information, RWTH Aachen University, Aachen D-52056, Germany \\ ${ }^{4}$ Peter Grünberg Institute, Theoretical Nanoelectronics, Forschungszentrum Jülich, Jülich D-52425, Germany
}

(Received 3 November 2020; accepted 5 March 2021; published 8 April 2021)

\begin{abstract}
Ensuring the correct functioning of quantum error correction (QEC) circuits is crucial to achieve fault tolerance in realistic quantum processors subjected to noise. The first checkpoint for a fully operational QEC circuit is to create genuine multipartite entanglement (GME) across all subsystems of physical qubits. We introduce a conditional witnessing technique to certify GME that is efficient in the number of subsystems and, importantly, robust against experimental noise and imperfections. Specifically, we prove that the detection of entanglement in a linear number of bipartitions by a number of measurements that also scales linearly, suffices to certify GME. Moreover, our method goes beyond the standard procedure of separating the state from the convex hull of biseparable states, yielding an improved finesse and robustness compared to previous techniques. We apply our method to the noisy readout of stabilizer operators of the distance-three topological color code and its flag-based fault-tolerant version. In particular, we subject the circuits to combinations of three types of noise, namely, uniform depolarizing noise, two-qubit gate depolarizing noise, and bit-flip measurement noise. We numerically compare our method with the standard, yet generally inefficient, fidelity test and to a pair of efficient witnesses, verifying the increased robustness of our method. Last but not least, we provide the full translation of our analysis to a trapped-ion native gate set that makes it suitable for experimental applications.
\end{abstract}

DOI: 10.1103/PRXQuantum.2.020304

\section{INTRODUCTION}

The continued effort to control quantum systems with ever increasing accuracy has led to various quantum processors [1], comprising tens of qubits and, more recently, to the demonstration of quantum supremacy [2]. However, in the mid and long term, an important goal towards real-world applications of quantum computation is to demonstrate fault tolerance (FT) with low resources [3,4]. Quantum error correction (QEC) [5-7] is a building block of the FT theory $[8,9]$ the purpose of which is to protect the encoded information from the detrimental accumulation of errors by detecting and correcting them along with the computation. A current goal in this direction is to reach the break-even point where FTQEC demonstrates advantage over computations with bare physical qubits. An initial

\footnotetext{
*andrer22@ucm.es
}

Published by the American Physical Society under the terms of the Creative Commons Attribution 4.0 International license. Further distribution of this work must maintain attribution to the author(s) and the published article's title, journal citation, and DOI. proposal to attest this benchmark in near-term devices was to compare the performance of a family of QEC circuits acting on logical qubits with the best-possible counterparts using unencoded physical qubits [3]. We note, however, that any attempt towards the rigorous demonstration of FT must first ensure that QEC circuits function correctly.

While physical qubits and gates can be characterized using different techniques, e.g., spectroscopic and interferometric methods extracting the coherence times [10], and randomized benchmarking or process tomography characterizing gate performances [11,12], an efficient characterization for medium-sized registers and circuits that implement QEC protocols is still lacking. Simulating such circuits for general noise and nontrivial-size encondings to demonstrate this break-even point is computationally very demanding, even in the light of the recent result by Beale and Wallman [13] wherein the computational cost of effective-logical-noise simulation is significantly reduced. Alternative milder criteria for a quantitative evaluation of the performance of QEC circuits show that the requirements to demonstrate beneficial QEC are in the reach of near-future quantum platforms, e.g., using trapped-ion quantum processors $[4,14,15]$. Nonetheless, prior to the 
development of such optimal full codes for QEC, which must function in the vicinity of FT thresholds, it would be desirable to leverage the currently available noisier devices, e.g., by analyzing the performance of building blocks of QEC codes.

To achieve this goal, we note that the capacity to generate entanglement lies at the very heart of QEC protocols, since preparation circuits of encoded logical states [16,17], and syndrome readout schemes [14,18,19], generate genuinely multipartite entangled states. On the same note, the necessity of unbounded genuine multipartite entanglement (GME) to obtain an advantage in any quantum-computation algorithm was noted by Jozsa and Linden [20]. As a result, certifying GME serves as a partial benchmark in the progress towards FT quantum computation with noisy quantum processors by proving the genuine quantum-mechanical nature and functioning of the building blocks of larger QEC circuits in terms of their power to generate GME.

In general, verifying GME is a daunting task, which can be understood from the following four points. Theoretically, GME detection requires certifying entanglement within all possible bipartitions of a multipartite system. The number of bipartitions, however, grows exponentially with the number of subsystems. This implies that, in general, the required number of bipartite entanglement tests increases exponentially with the addition of every subsystem. Furthermore, certifying bipartite entanglement in its own is known to be NP-hard [21,22]. From an experimental perspective, not every theoretically conceived measurement can be implemented in practice, calling for the compliance of entanglement certification methods with such limitations. In addition, experiments suffer from imperfections that may reduce the capabilities of naive theoretical schemes of entanglement detection considerably. It is thus necessary to devise and continuously improve efficient GME certification methods.

An elegant approach that addresses these four challenges to a reasonable extent is entanglement witnessing [23-28]. An entanglement witness is an observable for which a negative expectation value in a given state indicates that the state is necessarily entangled. Interestingly, entanglement witnesses can be constructed from local and experimentally friendly observables $[29,30]$, and further optimized to show some degree of noise resilience [25,31-33]. In particular, the guaranteed existence of a witness for each entangled state makes witnesses extremely useful in experiments [23]. Nevertheless, in multipartite scenarios, there seems to be a trade-off between the finesse of entanglement witnesses and the number of measurements needed to carry them out. Specifically, as discussed in detail below, the standard method of GME certification using a single witness [26,29], reduces the exponential growth of the required number of bipartitions and measurements to a linear one by trading the individual bipartitions for the convex hull of all biseparable states, and detecting those states outside this convex hull. This advantage comes at a price, namely, a significant reduction in the robustness of the witness against experimental imperfections.

In this work, we introduce conditional entanglement witnessing, a witnessing technique that combines ideas from localizable entanglement [34] with entanglement witnessing, in order to reduce the exponential complexity of the GME detection to a linear number of tests while, at the same time, achieving a remarkable robustness against noise and errors. Our approach facilitates the detection of GME in quantum states that, although lying within the convex hull of biseparable states, remain masked to the single-witness approach.

We prove that the separation between any quantum state and only a linear number of conditional biseparable sets suffices for the purpose of certifying GME in that particular state. Due to the fact that for each individual bipartition the corresponding set of biseparable states is delimited closely by the convex set of conditional biseparable states, our conditional witnesses allow for a finer state discrimination between GME and biseparable states even in the presence of noise and experimental imperfections. We show the efficiency and robustness of conditional witnessing by applying it to the output of non-FT and FT stabilizer measurement circuits of a $d=3$ topological QEC color code [35] subjected to phenomenological, circuit-level, and measurement noise models. We then numerically compare the performance of our method against the standard witnessing methods. Our results demonstrate that conditional witnessing is simultaneously more efficient and more robust in proving GME in noisy quantum systems.

The paper is organized as follows. In Sec. II, we give an overview of the stabilizer formalism for the topological QEC color code, together with the flag-based FTQEC scheme. Section III discusses ideal non-FT and FTQEC circuits. In Sec. IV, multipartite entanglement and its standard witnessing are reviewed. We then introduce our method of conditional entanglement witnessing. Section $\mathrm{V}$ is devoted to the compilation of the QEC circuits into the native trapped-ion gates. Next, phenomenological, circuitlevel, and measurement error models used in the simulations are introduced. In Sec. VI we present our numerical results for our conditional witnessing technique and some of the best standard witnessing methods available applied to noisy trapped-ion platforms. We discuss the robustness of the methods based on results obtained for different noise channels applied to non-FT and flag-based FT trapped-ion circuits. Finally, in Sec. VII we provide the conclusions and outlook of our work.

\section{FLAG-QUBIT-BASED PROTOCOL FOR FAULT-TOLERANT QEC}

QEC promises to battle environmental decoherence and experimental imperfections during a quantum computation 
by redundantly encoding quantum information in multipartite logical states [36]. In order to protect the information that is stored redundantly, active QEC considers performing certain types of measurements frequently to detect and correct the error that has corrupted the state without affecting the encoded information [5-7]. Many leading QEC codes can be described within the stabilizer formalism [37].

A $[(n, k, d)]$ stabilizer quantum code, which encodes $k$ logical qubits into $n$ physical qubits with a code distance $d$, can correct up to $t=\lfloor(d-1) / 2\rfloor$ errors. The stabilizers $S_{i}$ form an Abelian subgroup $\mathcal{S}$ of size $|\mathcal{S}|=2^{G}$ of the $n$-qubit Pauli group $\mathcal{P}_{n}=\{ \pm 1, \pm i\} \times\{I, X, Y, Z\}^{\otimes n}$ where $I, X, Y, Z$ are the single-qubit Pauli matrices. Such a subgroup is typically specified via a generating subset of $G$ linearly independent and mutually commuting Pauli operators, $g:=\left\{g_{i}\right\}_{i=1}^{G}$, that are known as parity checks-or subgroup generators - so that $\mathcal{S}=\left\langle g_{1}, \ldots, g_{G}\right\rangle$. The latter means that any element of the stabilizer subgroup $S \in \mathcal{S}$ can be obtained as a specific product of the generators of the form

$$
S=\bigotimes_{i} g_{i} \text { with } g_{i} \in g
$$

The measurement of a single stabilizer yields one of its two possible eigenvalues $\lambda= \pm 1$, which is $2^{n-1}$-fold degenerate. Since the parity checks form a set of compatible observables, it is possible to unambiguously specify a $2^{n-G}$-dimensional subspace of the $n$-qubit state space through their common eigenspace with eigenvalue +1 ,

$$
\mathcal{L}=\left\{|\psi\rangle \in \mathbb{C}^{2 n}: S|\psi\rangle=|\psi\rangle, \forall S \in \mathcal{S}\right\}
$$

The subspace $\mathcal{L}$ is known as the code space, which can be used to embed (codify) the $k=n-G$ logical qubits. The logical generators $\left\{X_{\ell}^{L}, Z_{\ell}^{L}\right\}_{\ell=1}^{k}$ are then elements of the Pauli group $\mathcal{P}_{n}$ that commute with all stabilizers $S \in \mathcal{S}$, but which are not stabilizers themselves. Defining the centralizer of $\mathcal{S}$ in $\mathcal{P}_{n}$ as $\mathcal{C}(\mathcal{S})=\left\{P \in \mathcal{P}_{n}: P S=S P, \forall S \in \mathcal{S}\right\}$, logical operators lie within $\mathcal{C}(\mathcal{S}) \backslash \mathcal{S}$. The key idea here is that the logical operators transform the logical state within the code space while leaving the code space as a whole invariant.

It is central to the construction of a QEC code using the stabilizer formalism that the measurements of parity checks allow us to extract the so-called error syndrome without altering the stored quantum information. This typically requires the use of ancillary qubits into which the error syndrome is mapped through a sequence of entangling gates followed by a projective measurement on them. In practice, however, the syndrome measurements will also be affected by decoherence and noise, thus introducing further errors. This raises a crucial aspect of QEC, namely, the notion of fault tolerance [38] - the circuits used for the

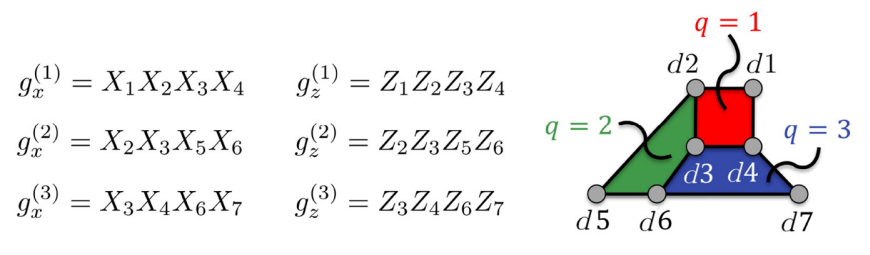

FIG. 1. The seven-qubit color code arranged in a threecolorable planar lattice with qubits lying on the vertices, and two types of parity checks per plaquette, hence $G=6$. This code yields a single logical qubit, $k=1$, encoded in $n=7$ data qubits, distance $d=3$, and can correct for $t=1$ error.

syndrome readout and other operations must be designed in such a way that errors do not proliferate in an uncontrolled manner, cascading into multiple errors that would affect larger parts of the quantum register. In other words, the QEC code should cope with errors stemming from both the quantum computation and the syndrome extraction even if the measurement process is faulty. Such FT circuit constructions are essential to harness the full potential correcting power of the QEC code under consideration.

One of the most attractive strategies for active QEC is based on topological codes where the physical qubits are arranged on planar lattices and the stabilizers have a local support, i.e., they involve only neighboring qubits in the planar lattice [36]. In contrast to QEC codes based on concatenation [7], the syndrome extraction for topological codes requires only local measurements simplifying in this way the experimental implementation of rounds of QEC, i.e., syndrome extraction followed by the correction of the most-likely error. For instance, for topological color codes [35], physical qubits are arranged on the vertices of a trivalent three-colorable planar lattice (see Fig. 1). The parity-check operators are the following pair of operators ( $X$ - and $Z$-type parity checks) per plaquette $q$ of the lattice, and have a local support on the qubits located at the vertices of such plaquette

$$
g_{x}^{(q)}=\bigotimes_{i \in v(q)} X_{i}, \quad g_{z}^{(q)}=\bigotimes_{i \in v(q)} Z_{i},
$$

where $v(q)$ is the set of vertices that belong to plaquette $q$. Accordingly, there are $G=6$ parity checks for the smallest color code with $n=7$ physical qubits each acting locally on only four qubits so that the code space is two-dimensional encoding $k=n-G=1$ logical qubit.

To detect the errors that may have corrupted the logical qubit, one must measure all four-qubit parity checks using a FT scheme and infer the location of the most-likely error from the classical information obtained from the sequence of \pm 1 parity-check outcomes known as the error syndrome. In this particular example, the parity-check information is extracted via projective measurements on an ancillary syndrome qubit. In Fig. 2(a), we show a circuit for the readout of an $X$-type parity check of a single 

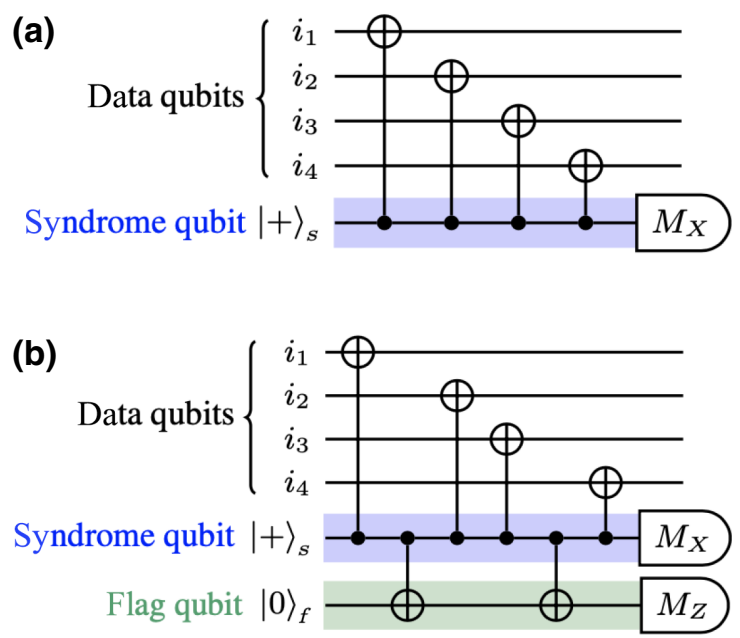

FIG. 2. Error syndrome extraction circuits in the color code for (a) the non-FT and (b) the flag-based FT readouts of the $X$ type parity check $g_{x}^{(q)}$ in Eq. (3) of any plaquette $q$ in Fig. 1. The physical and data qubits correspond to the corresponding four physical qubits of this plaquette with indexes $i_{1}, i_{2}, i_{3}, i_{4} \in$ $\{d 1, d 2, d 3, d 4, d 5, d 6, d 7\}$. These qubits are sequentially coupled via CNOT entangling gates to the syndrome qubit, which must be initialized in a specific state, and then measured in the corresponding basis. In the flag-based scheme, an additional ancillary qubit is appropriately initialized, coupled, and measured to reveal crucial information to attain FT.

plaquette of the seven-qubit color code. We note, however, that since all four plaquette qubits are coupled to the same ancillary qubit through the sequential two-qubit gates, single-qubit errors can proliferate and create errors with a larger support. Following the syndrome measurement approach using a single ancilla, any such error will result in a logical error, thereby violating the FT design principle.

A recent proposal for an efficient recovery of FT during the syndrome extraction is the flag-based stabilizer readout [39-41]. As shown in Fig. 2(b), this technique uses a single additional ancillary qubit, the flag qubit, which is coupled to the syndrome qubit. We note that, in general, even if more flag qubits are required for largerdistance codes [40], these are not required to be initially prepared in a multipartite entangled Greenberger-HorneZeilinger (GHZ) [42] state, which must also be verified to avoid a non-FT proliferation of errors $[43,44]$. This brings about significant advantage compared to cat-state ancillary methods $[43,44]$. In the case of the seven-qubit color code, as shown in Fig. 2(b), the flag qubit is coupled to the syndrome qubit to detect whether or not multiple errors might have cascaded onto the data qubits. We note that the flag qubit by itself does not suffice to correct for such higher-weight errors. However, when combined with the subsequent parity-check measurements using the syndrome qubit, it can be used to unequivocally identify and correct these dangerous errors achieving the desired fault tolerance $[39,45]$.

\section{IDEAL PLAQUETTE CIRCUIT}

We are interested in evaluating the correct functioning of the plaquette readout circuits, as they are the smallest building blocks of the circuitry for the topological color code [35]. To do that, it is useful to first work out the details of the ideal circuits shown in Fig. 2. Here, by ideal we mean circuits that are free from state preparation and measurement (SPAM) and gate errors. This is done in the following section. We emphasize that, here, we focus only on the $g_{x}$ generator. Nevertheless, a similar procedure can be implemented using the $g_{z}$ generator as presented in Appendix A.

\section{A. Ideal non-FT plaquette circuit}

Let us start our analysis with the simplest example of the ideal plaquette circuit in Fig. 2(a). In a full QEC code, the syndrome qubit can be prepared in $|+\rangle_{s}=\left(|0\rangle_{s}+\right.$ $\left.|1\rangle_{s}\right) / \sqrt{2}$, while the data qubits $\left\{i_{1}, i_{2}, i_{3}, i_{4}\right\}$ are, in general, part of a larger logical state. After applying the circuit, the syndrome qubit would contain the $Z$-type error information. This information could then be extracted via a projective measurement of the syndrome qubit. In order to evaluate the performance of the color-code blocks, however, we do not need to read out the syndrome. In this case, our interest lies in the characterization of the corresponding circuit from the perspective of GME. To do so, it turns out to be sufficient to assume that data qubits are initialized in $|0\rangle^{\otimes 4}$. Next, four CNOT gates are sequentially applied with the syndrome as the common control qubit while the data qubits play the role of the corresponding targets. Recall that a CNOT gate between the control and the target qubits, $c$ and $t$, respectively, can be written in terms of Pauli operators as $\mathrm{CNOT}_{c, t}=\left\{\left(I_{c}+Z_{c}\right) I_{t}+\left(I_{c}-Z_{c}\right) X_{t}\right\} / 2$. After some algebra, the output state obtained is

$$
\left|\psi_{\text {out }}\right\rangle=\frac{|0\rangle^{\otimes 5}+|1\rangle^{\otimes 5}}{\sqrt{2}}=\left|\mathrm{GHZ}_{+}^{\otimes 5}\right\rangle
$$

The resulting five-qubit GHZ state of Eq. (4) can be understood as the one-dimensional code space of the stabilizer subgroup

$$
\begin{aligned}
\mathcal{S}_{5 q}^{\mathrm{GHZ}} & =\left\langle g_{1}=Z_{1} Z_{2}, g_{2}=Z_{2} Z_{3}, g_{3}=Z_{3} Z_{4}, g_{4}=Z_{4} Z_{5}, g_{5}\right. \\
& \left.=X_{1} X_{2} X_{3} X_{4} X_{5}\right\rangle
\end{aligned}
$$


and thus, it can be recast as the product of the projections onto the common +1 eigenspace of $\mathcal{S}_{5 q}$, namely

$$
\varrho_{\text {out }}=\left|\mathrm{GHZ}_{+}^{\otimes 5}\right\rangle\left\langle\mathrm{GHZ}_{+}^{\otimes 5}\right|=\prod_{i=1}^{5}\left(\frac{I_{i}+g_{i}}{2}\right)=\frac{1}{2^{5}} \sum_{i=1}^{2^{5}} S_{i} .
$$

Let us now briefly analyze the effect of a dangerous error in the syndrome qubit. Consider, for example, the case wherein the syndrome suffers from a Pauli- $X$ error between the second and third CNOT gates; see Fig. 3(a). Using the standard propagation of errors across CNOT gates [8], it is easy to calculate the output state as

$$
\left|\psi_{\text {out }}\right\rangle=X_{S} X_{3} X_{4}\left|\mathrm{GHZ}_{+}^{\otimes 5}\right\rangle=X_{1} X_{2}\left|\mathrm{GHZ}_{+}^{\otimes 5}\right\rangle \text {. }
$$

It is evident from Eq. (7) that the syndrome qubit carries no information about the error and that the single-qubit error has turned into a weight- 2 error showcasing the non-FT aspect of this circuit. Importantly, even in the case of a coherent rotation error $\exp (i \phi X)$ of the syndrome qubit, as depicted in Fig. 3(b), a simple calculation results in the output state

$$
\left|\psi_{\text {out }}\right\rangle=\cos \phi\left|\mathrm{GHZ}_{+}^{\otimes 5}\right\rangle+\mathrm{i} \sin \phi X_{S} X_{3} X_{4}\left|\mathrm{GHZ}_{+}^{\otimes 5}\right\rangle,
$$

which again represents the fact that a projective $X$ measurement of the syndrome qubit does not reveal the error. This brings us to the FT scheme of the next section.

\section{B. Ideal flag-based plaquette circuit}

We now consider the FT flag-based circuit presented in Fig. 2(b). Similar to the noiseless non-FT plaquette circuit of Sec. III A, we assume the syndrome qubit is initialized in the state $|+\rangle_{s}$ and the data qubits $\left\{i_{1}, i_{2}, i_{3}, i_{4}\right\}$ in the product state $|0\rangle^{\otimes 4}$. The flag qubit, on the other hand, is prepared in $|0\rangle_{f}$. The main difference between the flag-based and the non-FT circuits is that the two additional CNOT gates between the syndrome and the flag propagate the potential error in the syndrome qubit to the flag qubit while this does not corrupt the resulting state in the event of an ideal errorfree realization. This enables one to infer about the error in the syndrome qubit that propagates to multiple errors on the data by a projective measurement of the flag qubit. When there are no errors in the syndrome, it is expected from the ideal circuit to yield the output state

$$
\left|\psi_{\text {out }}\right\rangle=\frac{\left(|0\rangle^{\otimes 5}+|1\rangle^{\otimes 5}\right)|0\rangle_{f}}{\sqrt{2}}=\left|\mathrm{GHZ}_{+}^{\otimes 5}\right\rangle|0\rangle_{f} .
$$

However, if the syndrome qubit suffers from a single Pauli$X$ error happening between the two CNOTs, as shown in
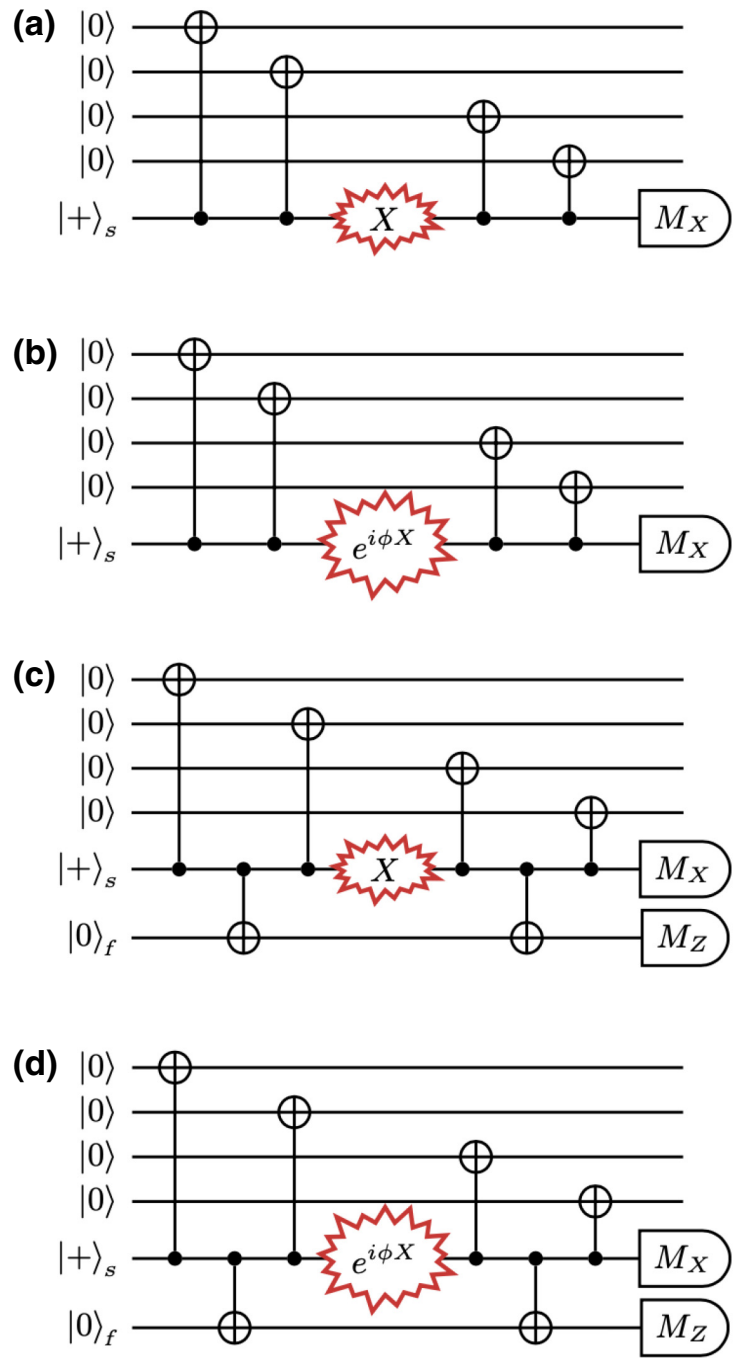

FIG. 3. Introduction of dangerous errors in noisy plaquette circuits for (a),(b) the non-FT and (c),(d) the flag-based FT readouts of the $g_{x}$ generator. (a) A single Pauli- $X$ error occurring on the syndrome qubit in a dangerous position leads to a cascade of errors in the data qubits. (b) Coherent-rotation errors in the syndrome qubit also lead to multiple undetectable errors in the data qubits. In (c), in contrast to the non-FT cases, outcomes of the measurement on the flag qubit reveals the cascade of errors from the syndrome qubit to the data. Similarly in (d), the coherentrotation error is detected through measurement outcomes of the flag qubit.

Fig. 3(c), it will propagate to the flag qubit as a bit flip. This is reflected in the output state

$$
\left|\psi_{\text {out }}\right\rangle=X_{s} X_{3} X_{4}\left|\mathrm{GHZ}_{+}^{\otimes 5}\right\rangle|1\rangle_{f} .
$$

As a result, in contrast to the non-FT scenario of Sec. III A, the -1 measurement outcome of the flag indicates the propagation of a correlated weight- 2 error in the syndrome and data. Note that there are other events in a noisy experimental realization of the circuit, for example, a measurement error on the flag qubit, which could also trigger 
the flag. Therefore, to construct one FTQEC cycle, further measurements of the stabilizers would be required to discriminate between these cases [45].

Finally, if the syndrome qubit suffers from the coherentrotation error $\exp (i \phi X)$ between the second and third syndrome-data CNOT gates shown in Fig. 3(d), then the output state of the ideal circuit becomes

$$
\begin{aligned}
\left|\psi_{\text {out }}\right\rangle= & \cos \phi|0\rangle_{f}\left|\mathrm{GHZ}_{+}^{\otimes 5}\right\rangle \\
& +\mathrm{i} \sin \phi|1\rangle_{f} X_{s} X_{3} X_{4}\left|\mathrm{GHZ}_{+}^{\otimes 5}\right\rangle .
\end{aligned}
$$

Here again, the flag unveils a cascaded error in the syndrome and data qubits.

Having understood the working principles and the ideal outputs of each plaquette in both non-FT and flag-based FT scenarios, we are ready to introduce our main tool for characterizing the performance of these circuits, namely, the conditional entanglement witnessing.

\section{CONDITIONAL ENTANGLEMENT WITNESSING}

\section{A. Multipartite entanglement and its witnesses}

In this section we review the well-established theory of multipartite entanglement witnessing $[26,46]$. The readers familiar with these details might skip this section and move on to Sec. IV B. We identify the subsystems of an $n$-partite system using an index set $\mathbf{I}:=\{1,2, \ldots, n\}$ so that, given the Hilbert space $\mathcal{H}_{i}$ associated with each subsystem, the Hilbert space of the total system is given by $\mathcal{H}_{\mathbf{I}}=\otimes_{i \in \mathbf{I}} \mathcal{H}_{i}$. Evidently, it is possible to group the subsystems in many different ways. Calling each possible grouping a partitioning, they correspond to partitionings of the index set $\mathbf{I}$. An $m$ partitioning $\left(\mathbf{I}_{1}\left|\mathbf{I}_{2}\right| \cdots \mid \mathbf{I}_{m}\right)$ is thus a specific grouping of subsystems such that $\cup_{x} \mathbf{I}_{x}=\mathbf{I}$ and $\mathbf{I}_{x} \cap \mathbf{I}_{y}=\emptyset$ for any $x \neq y$. It is customary to call two partitionings bipartitions. We also denote the Hilbert space associated with each party by $\mathcal{H}_{\mathbf{I}_{j}}=\otimes_{x \in \mathbf{I}_{j}} \mathcal{H}_{x}$.

Recall that each quantum system is described by a density operator $\varrho$, which is a unit-trace positive member of the set of all bounded linear transformations $\mathcal{L} \operatorname{in}(\mathcal{H})$ on the Hilbert space $\mathcal{H}$ assigned to the system. The set of all quantum states of a system is denoted by $\mathcal{S} t \subset \mathcal{L} \operatorname{in}(\mathcal{H})$, which is a compact convex set. Applying these rules to the multipartite scenario for a presumed $m$ partitioning $\left(\mathbf{I}_{1}|\cdots| \mathbf{I}_{m}\right)$, the state space of each party $\mathbf{I}_{j}$ is denoted by $\mathcal{S} t_{\mathbf{I}_{j}} \subset \mathcal{L} \operatorname{in}\left(\mathcal{H}_{\mathbf{I}_{j}}\right)$, while the state space of the total system is identified as $\mathcal{S} t_{\mathbf{I}} \subset \mathcal{L} \operatorname{in}\left(\mathcal{H}_{\mathbf{I}}\right)$. Now, imagine that parties in $\left(\mathbf{I}_{1}|\cdots| \mathbf{I}_{m}\right)$ are spatially separated and all they can do is perform local operations on their possessed quantum systems and possibly communicate classical messages, known as the local operation and classical communication
(LOCC) paradigm, and ask what quantum states of the total system can the parties prepare. It can be shown that the quantum states that the parties can achieve in this setting are of the form [47]:

$$
\varrho=\sum_{i} p_{i} \varrho_{\mathbf{I}_{1} ; i} \otimes \cdots \otimes \varrho_{\mathbf{I}_{m} ; i}
$$

wherein $\varrho_{\mathbf{I}_{x} ; i} \in \mathcal{S} t_{\mathbf{I}_{x}}$ for any $x \in\{1, \ldots, m\}$ and $\left\{p_{i}\right\}$ is a probability distribution. The quantum states of this form are called $m$-separable states with respect to the partitioning $\left(\mathbf{I}_{1}|\cdots| \mathbf{I}_{m}\right)$ and their collection forms a closed convex set denoted by $\mathcal{S e p}_{\mathbf{I}_{1}|\cdots| \mathbf{I}_{m}}$. The set of $m$-separable states for any given partitioning is also dense and convex. Most importantly, however, is that $\mathcal{S e p}_{\mathbf{I}_{1}|\cdots| \mathbf{I}_{m}} \subsetneq \mathcal{S} t_{\mathbf{I}}$, i.e., there are quantum states of the total system that cannot be prepared by the $m$ parties using LOCC. Such states are called entangled with respect to the $m$ partitioning $\left(\mathbf{I}_{1}|\cdots| \mathbf{I}_{m}\right)$.

Entanglement in an $n$-partite system has a complex structure because the possibilities of choosing the partitioning grow exponentially with the number of subsystems $[26,48]$. A particularly interesting class of multipartite entangled states, however, is the one formed by states that are entangled within all possible bipartitions. These states are called genuinely multipartite entangled (GME), or-sometimes - fully inseparable [46,49]. Henceforth we use the former name for such states. This is the strongest form of multipartite entanglement as it can be shown to imply entanglement within all possible $k$ partitionings of the system $[26,48]$.

Let us remark that the certification of GME in an $n$-partite system requires verification of entanglement in $N_{b}=(1 / 2) \sum_{i=1}^{n-1}\left(\begin{array}{l}n \\ i\end{array}\right)=2^{n-1}-1$ different bipartitions, showing the exponential increase in both analytical and practical complexity of this task. Certifying entanglement of an arbitrary quantum state, be it bipartite or multipartite, is also known to be very hard from the perspective of complexity theory [21,22]. This is because determining whether a given quantum state is entangled or not is equivalent to deciding whether it is inside or outside of a suitable convex set of separable states. However, any such set is not a simplex, meaning that it does not have a finite number of "straight border lines" to be checked for the separation of the state under consideration [50]. Nevertheless, it is possible to obtain many sufficient (but maybe not necessary) conditions for entanglement of quantum states [27], a very large class of which are entanglement witnesses [23-25,51].

Simply speaking, an entanglement witness is a quantum observable whose expectation value is bounded for all separable states. This bound, however, can be violated by suitable entangled states. More precisely, for every entangled state with respect to a specific $m$ partitioning $\left(\mathbf{I}_{1}|\cdots| \mathbf{I}_{m}\right)$, $\varrho \notin \mathcal{S e p}_{\mathbf{I}_{1}|\cdots| \mathbf{I}_{m}}$, there exists a bounded Hermitian operator 
$W_{\mathbf{I}_{1}|\cdots| \mathbf{I}_{m}}$ such that

$$
\begin{aligned}
\forall \sigma \in \mathcal{S e p}_{\mathbf{I}_{1}\left|\mathbf{I}_{2}\right| \cdots \mid \mathbf{I}_{m}} & \operatorname{Tr}\left(W_{\mathbf{I}_{1}|\cdots| \mathbf{I}_{m}} \sigma\right) \geq 0 \\
& \operatorname{Tr}\left(W_{\mathbf{I}_{1}|\cdots| \mathbf{I}_{m}} \varrho\right)<0 .
\end{aligned}
$$

Importantly, the existence of the witness operator $W_{\mathbf{I}_{1}|\ldots| \mathbf{I}_{m}}$ is guaranteed by the Hahn-Banach separation theorem [51]. Finding a witness operator with properties in Eq. (13) may seem to be difficult. Thankfully, using the affine properties of the space of bounded linear operators on Hilbert spaces, it was shown $[25,26,51,52]$ that every entanglement witness can be constructed from a bounded linear test operator, henceforth denoted by $L$, so that

$$
W_{\mathbf{I}_{1}|\cdots| \mathbf{I}_{m}}=l_{\mathbf{I}_{1}|\cdots| \mathbf{I}_{m}} I-L_{\mathbf{I}_{1}|\cdots| \mathbf{I}_{m}},
$$

in which the so-called separability bound is

$$
l_{\mathbf{I}_{1}|\cdots| \mathbf{I}_{m}}=\sup _{\sigma \in \mathcal{S e p}_{\mathbf{I}_{1}|\cdots| \mathbf{I}_{m}}} \operatorname{Tr}\left(L_{\mathbf{I}_{1}|\cdots| \mathbf{I}_{m}} \sigma\right)
$$

While the optimization in Eq. (15) seems demanding, it is known that entanglement witnessing is efficiently decidable $[53,54]$. Now, according to Eq. (13), any state $\varrho$ for which $\operatorname{Tr}\left(L_{\mathbf{I}_{1}|\cdots| \mathbf{I}_{m}} \varrho\right)>l_{\mathbf{I}_{1}|\cdots| \mathbf{I}_{m}}$ is entangled with respect to the partitioning $\left(\mathbf{I}_{1}|\cdots| \mathbf{I}_{m}\right)$.

The program of entanglement witnessing thus proceeds as follows. It is assumed that some information about the entangled state to be detected is available a priori, e.g., the quantum state that results from an ideal noiseless circuit is known. The goal is to devise an experimentally friendly observable, i.e., one that can be measured with relatively low cost, for instance, in the number of settings, based on the given information, which is also resilient against the state preparation and measurement imperfections. We note that, in general, different witnesses are required for different partitionings [26,48]. In this paper, however, we are interested in GME witnessing and shall thus focus on bipartitions.

Let us give a simple example of how the standard GME witnessing can be performed. Consider the circuit shown in the top panel of Fig. 2. We show in Sec. III A that the ideal output of this circuit is the five-qubit GHZ state,

$$
\left|\psi_{\text {out }}\right\rangle=\left|\mathrm{GHZ}_{+}^{\otimes 5}\right\rangle=\frac{|0\rangle^{\otimes 5}+|1\rangle^{\otimes 5}}{\sqrt{2}} .
$$

To show that this state is genuinely multipartite entangled, we have to check the entanglement in all $N_{b}=15$ possible bipartitions. Let us focus on one of them, say $(s \mid 1,2,3,4)$ where we use the symbol $s$ for the the syndrome qubit. It is easy to see that, with respect to this bipartition, the GHZ state is equivalent to a bipartite Bell state, i.e.,

$$
\left|\psi_{\text {out }}\right\rangle=\left|\mathrm{GHZ}_{+}^{\otimes 5}\right\rangle=\frac{|0 \overline{0}\rangle+|1 \overline{1}\rangle}{\sqrt{2}}
$$

where $|\overline{0}\rangle=|0\rangle^{\otimes 4}$ and $|\overline{1}\rangle=|1\rangle^{\otimes 4}$. Consider now the test operator

$$
L=\frac{(|0 \overline{0}\rangle+|1 \overline{1}\rangle)(\langle 0 \overline{0}|+\langle 1 \overline{1}|)}{2}=\left|\mathrm{GHZ}_{+}^{\otimes 5}\right\rangle\left\langle\mathrm{GHZ}_{+}^{\otimes 5}\right| .
$$

Using the similarity with the Bell state it is easy to compute the supremum expectation value of this test operator over all biseparable states $\mathcal{S e p} p_{s \mid 1,2,3,4}$ obtaining the separability bound of Eq. (15) with respect to this bipartition as

$$
l_{s \mid 1,2,3,4}=\sup _{\sigma \in \mathcal{S} \operatorname{Sep}_{s \mid 1,2,3,4}} \operatorname{Tr}(L \sigma)=\frac{1}{2} .
$$

Consequently, one can define an entanglement witness with respect to this bipartition as

$$
W_{s \mid 1,2,3,4}=\frac{1}{2} I-L,
$$

so that $\operatorname{Tr}\left(W_{s \mid 1,2,3,4} \sigma\right) \geq 0$ for all biseparable states $\sigma \in$ $\mathcal{S e p}_{s \mid 1,2,3,4}$, while there exist quantum states for which

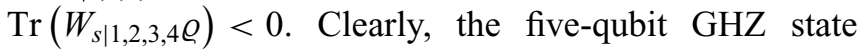
itself is an example of the latter. It then follows that $\left|\mathrm{GHZ}_{+}^{\otimes 5}\right\rangle\left\langle\mathrm{GHZ}_{+}^{\otimes 5}\right| \notin \mathcal{S} e p_{s \mid 1,2,3,4}$, which must be read as "the five-qubit GHZ state is entangled with respect to the bipartition $(s \mid 1,2,3,4)$." For this example, due to the symmetries of the test operator $L$ in Eq. (18) with respect to different bipartitions, it is easy to show that the maximum expectation value of the test operator for all bipartitions is indeed the same and equal to $1 / 2$. As a result, we find that the witness operator of Eq. (20) is in fact capable of detecting the entanglement of the five-qubit GHZ state within all possible bipartitions and thus serves as a faithful witness of GME.

We emphasize, however, that this is not generally the case for arbitrary quantum states, generic test operators, and arbitrary bipartitions. In the most general case of certifying entanglement using a single test operator, a given quantum state is GME if the expectation value of the test operator for that state is larger than the maximum of the separability bounds Eq. (15) for all bipartitions [26], i.e.,

$$
\varrho \text { is GME if }\langle L\rangle=\operatorname{Tr}(L \varrho)>\max _{B \in \mathcal{B}} l_{B},
$$

where the maximum is taken over the set of all bipartitions $\mathcal{B}$, which has $|\mathcal{B}|=N_{b}$ elements. Now, suppose that $\varrho$ and $\sigma$ are two biseparable states with 


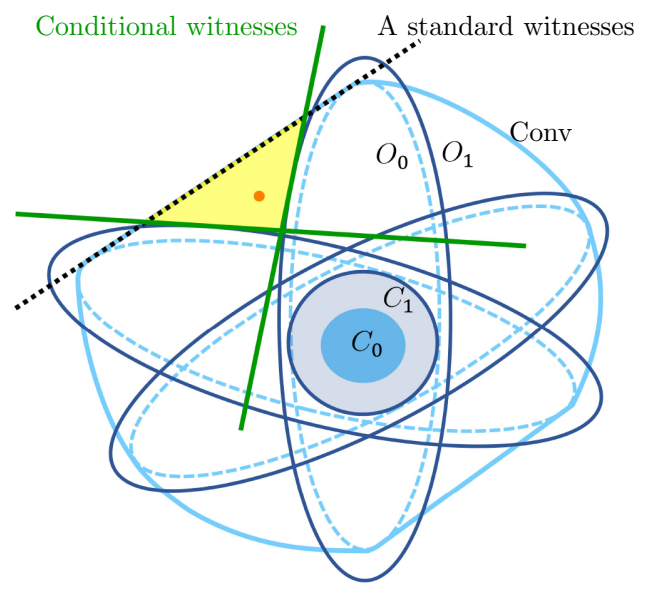

FIG. 4. Heuristic comparison between standard GME witnessing and conditional GME witnessing technique of Sec. IV C. The light-blue dashed ovals, say $O_{0}$, represent separable states with respect to a specific individual bipartition, also known as biseparable sets. Given $n$ subsystems there exist an exponential number, i.e., $N_{b}=2^{n-1}-1$, of biseparable sets. The closed convex hull of all biseparable sets is denoted as conv. The inner circle $C_{0}$ is the set of fully separable states. The dark-blue ovals, say $O_{1}$, represent the conditional biseparable states with respect to individual bipartitions, i.e., states for which entanglement cannot be localized between any two subsystems from parties of the bipartition. Evidently, $O_{0} \subsetneq O_{1}$. The circle $C_{1}$ represents the intersection of all conditional biseparable sets. The black dashed line represents a standard GME witness. It is clear that there exist quantum states, e.g., the red dot, that are GME and yet lie within the convex hull conv. Using Theorem 1 or Theorem 2 of Sec. IV B, it is enough to certify the entanglement of states with respect to only a linear number, i.e., $n-1$, of conditional bipartitions. We use $n-1$ witnesses (green solid lines) for this purpose. The intersection of detected states using such witnesses are also GME entangled states. Thus, the shaded yellow area highlights the advantage obtained using our conditional GME witnessing as discussed in Sec. IV C.

respect to two bipartitions $B_{1}$ and $B_{2}$, respectively. Thus, we have $\operatorname{Tr}(L \varrho) \leq \max _{B \in \mathcal{B}} l_{B}$ and $\operatorname{Tr}(L \sigma) \leq \max _{B \in \mathcal{B}} l_{B}$, which implies $\operatorname{Tr}\{L[p \varrho+(1-p) \sigma]\} \leq \max _{B \in \mathcal{B}} l_{B}, \forall p \in$ $[0,1]$. As this holds for any choice of $B_{1}$ and $B_{2}$, we conclude that the inequality $\langle L\rangle>\max _{B \in \mathcal{B}} l_{B}$ can be geometrically interpreted as a test that serves to identify points that lie outside the closed convex hull of all biseparable states. In other words, any standard GME witness using a single witness is only capable of detecting GME states that lie outside the closed convex hull of all biseparable states; see Fig. 4.

Nevertheless, an important aspect of the above construction is its potential experimental feasibility, depending on our choice of the test operator. First, to obtain the expectation value of the witness, it is enough to measure only the expectation value of the test operator. Second, the expectation value of the test operator of Eq. (18) can, in our particular case, be obtained by local measurements of the 32 stabilizers of the five-qubit GHZ state as given in Eq. (6). Hence, it is in practice possible to obtain the witness value and thus test the entangled nature of the state generated by the actual circuit. We note, however, that the number of stabilizers grows exponentially with the number of qubits, which makes this method practically challenging to implement. When using a single witness, this problem can cleverly be avoided by trading noise robustness for efficiency in the number of measurements as shown by Tóth and Gühne in Ref. [29]. We also emphasize that the latter difficulty must be distinguished from the obstacle due to the exponential growth in the number of bipartitions.

\section{B. Efficient certification of GME}

As we see, entanglement witnessing is meant to be an experimentally friendly technique to certify entanglement. Interestingly, there exist techniques to push witnessing to its limits so that a given witness delivers an impressive performance even in the presence of severe noise and errors $[25,31,32,55]$. In multipartite scenarios, however, we face a different problem, namely the scaling of the number of partitions with the input size, which seems to be persistent for any method of entanglement certification.

In the scenario of certifying GME that is relevant to our purpose an $n$-partite system contains $N_{b}=2^{n-1}-1$ bipartitions to be tested for entanglement the exponential growth of which is a major challenge for large systems. We note that, by choosing the witnessing method for entanglement certification and using the construction of Eqs. (19) and (20), one might construct $N_{b}$ witnesses for each bipartition using a single test operator $L$. Nevertheless, in general, there is an exponential number of optimizations as per Eq. (15) that must be performed [26]. One way to circumvent this practical limitation is to choose a test operator $L$ for which a suitable positive real number $l$ is known-by other means - such that $l$ is larger than the separability bound of Eq. (21) for all bipartitions. One such technique was adopted by Tóth and Gühne in Ref. [29]. However, the ease does not come for free: (i) the obtained witnesses are not the finest ones [25], as there may exist genuinely multipartite entangled states that cannot be detected by any such single witness, (ii) there is no general recipe for their construction, and (iii) they are limited to certain types of witnesses and systems as the value $l$ cannot be deduced for an arbitrary test operator without performing the optimizations.

In the following, we exploit the concept of localizable entanglement (LE) $[34,56]$ to introduce a general solution to this challenge that collapses the exponential complexity of GME witnessing to a linear growth for all system types in all dimensions. The price to pay, however, is that our technique provides only a sufficient condition for detecting GME, i.e., not every genuine multipartite entangled state can be detected using our approach. We emphasize, 
however, that for many operational scenarios including measurement-based quantum computation and quantum communication in which LE is a necessary resource [57, 58], our sufficient condition becomes necessary as well, hence providing a necessary and sufficient condition for practical purposes.

Let us begin with a simple example. Suppose that two parties Alice and Bob are holding quantum systems $A$ and $B$, respectively. In addition, Alice is holding an ancillary system $C$. Hence, we effectively have a tripartite system shared between two parties in the bipartition $(A C \mid B)$. We denote the quantum state of the total system with respect to this bipartition by $\varrho_{A C \mid B}$, and the amount of entanglement shared between Alice and Bob by $\mathcal{E}\left(\varrho_{A C \mid B}\right)$. We ask how that is related to the entanglement between subsystems $A$ and $B$. This is, in general, a difficult question to answer. Nevertheless, it is clear that if Alice performs a measurement on $C$ and, conditioned on the $i$ th outcome of this measurement, she finds out that the corresponding conditional state $\varrho_{A \mid B: i}$ is entangled, then the original state $\varrho_{A C \mid B}$ prior to the measurement must have been entangled with respect to the bipartition $(A C \mid B)$. The reason for this conclusion is that we know entanglement cannot increase via LOCC. If $\varrho_{A C \mid B}$ was to be separable with respect to the bipartition $(A C \mid B)$, then it would be impossible for Alice to create entanglement by a local measurement (i.e., her measurement on $C$ ) and classically communicating the result (i.e., her $i$ th outcome) to Bob. We thus see that entanglement between $A$ and $B$ conditioned on measurements on $C$ implies entanglement within the bipartition $(A C \mid B)$. Similarly, if we assume that Bob is holding the subsystem $C$, then we arrive at the conclusion that entanglement between $A$ and $B$ conditioned on measurements on $C$ also implies entanglement within the bipartition $(A \mid B C)$, hence the following lemma [59].

Lemma 1. Consider a tripartite state $\varrho_{A B C}$. Given that the conditional state $\varrho_{B \mid A: i}$ for the ith outcome of some measurement on the subsystem $C$ is entangled, this implies that $\varrho_{A B C}$ is entangled within both bipartitions $(A C \mid B)$ and $(A \mid B C)$.

The above example is reminiscent of the idea that, in a multipartite system, it might be possible to concentrate entanglement within a smaller set of subsystems by performing measurements on the remaining subsystems - and communicating the outcome - as first considered by Verstraete, Popp, and Cirac [34]. The maximum amount of entanglement that can be concentrated between Alice and Bob by such local measurements on average is dubbed as the "localizable entanglement." More precisely, suppose that $\mathcal{E}$ denotes an entanglement measure of quantum states that cannot be increased via LOCC. Given $n$ quantum systems in the state $\varrho$, the LE of the bipartition $\left(s_{x} \mid s_{y}\right)$ for $s_{x}, s_{y} \in\left\{s_{1}, \ldots, s_{n}\right\}$ after local measurements on subsystems $\left\{s_{x}, s_{y}\right\}^{\mathrm{c}}:=\left\{s_{1}, \ldots, s_{n}\right\} \backslash\left\{s_{x}, s_{y}\right\}$ with outcomes $\{i\}$ is given by

$$
\mathcal{L} \mathcal{E}_{x \mid y}(\varrho)=\sup _{\mathrm{M} \in \mathcal{C}} \sum_{i} p_{i} \mathcal{E}\left(\varrho_{x \mid y: i}\right)
$$

in which $\varrho_{x \mid y: i}$ is the joint conditional state of subsystems $s_{x}$ and $s_{y}$ given the outcome $i$, with probability $p_{i}$, of the local measurement $\mathrm{M}$ on subsystems $\left\{s_{x}, s_{y}\right\}^{\mathrm{c}}$. Moreover, the supremum is taken over the set $\mathcal{C}$ of all $(n-2)$-partite local measurements. For our example, it thus follows that $\mathcal{L E}_{A \mid B}>0$ implies entanglement in both bipartitions $(A C \mid B)$ and $(A \mid B C)$ as presented in Lemma 1.

Our main observation is that the implications of Lemma 1, in light of the concept of LE, can in fact be extended to any bipartition of any number of systems. Denote a generic bipartition of the $n$-partite system as $\left(s_{1}, \ldots, s_{j} \mid s_{j+1}, \ldots, s_{n}\right)$. According to Lemma 1, entanglement in this bipartition is certified once the entanglement between two subsystems $s_{x}$ and $s_{y}$ from each party, i.e., with $x \leq j$ and $y \geq j+1$, conditioned on appropriate measurement outcomes of the remaining $n-2$ subsystems, is verified. Naively, given the $j(n-j)$ choices of the pair $s_{x}$ and $s_{y}$ for each bipartition on top of the fact that there are yet $2^{n-1}-1$ bipartitions to be considered, there still seems to be an exponentially large number of entanglement certifications to be performed. Our central result stated in the following theorem shows that there is a huge redundancy in this process. Leveraging this redundancy allows us to reduce the exponential number of bipartitions to a linear one, hence making the GME detection efficient.

Theorem 1. Given an n-partite quantum system of any dimension, certification of entanglement between the subsystems $s_{1}$ and $s_{x}$ for all $s_{x} \in\left\{s_{1}\right\}^{c}$ conditioned on an outcome $i$ of suitable local measurements on the remaining $n-2$ subsystems is sufficient for the certification of GME of the system. The number of bipartitions needed to be checked is thus $n-1$.

Proof. To prove the theorem, let us first introduce a notation that simplifies and clarifies the procedure. For any pair of subsystems $s_{x}$ and $s_{y}$, on which we want to localize entanglement, we can denote by $\left(S_{L},\left[s_{x} \mid s_{y}\right], S_{R}\right)$ a bipartition with respect to which the entanglement is being certified. Here, $S_{L}$ and $S_{R}$ are the collection of all subsystems except $s_{x}$ and $s_{y}$ that belong to the left and right parties, respectively, and on which the conditioning takes place. We also call the block $\left[s_{x} \mid s_{y}\right]$ the conditional bipartition.

By using Lemma 1, for a fixed choice of subsystems $s_{x}$ and $s_{y}$, the localizable entanglement of any such bipartition is equivalent to that of the unique bipartition $\left(\left[s_{x} \mid s_{y}\right], S_{R}^{\prime}\right)$ where $S_{R}^{\prime}=S_{R} \cup S_{L}$. This follows directly by assuming that Alice is holding the subsystem $A=s_{x}$ while Bob holds 
$B=s_{y} \cup S_{L} \cup S_{R}$ and is able to make local measurements on $C=S_{R}^{\prime}$.

Thus, one can think of the fixed block $\left[s_{x} \mid s_{y}\right]$ sliding through to generate bipartitions of the form $\left(S_{L},\left[s_{x} \mid s_{y}\right], S_{R}\right)$ without changing the entanglement properties of the resulting bipartition. We symbolically denote this equivalence of bipartitions in terms of their localizable entanglement as $\left(\left[s_{x} \mid s_{y}\right], S_{R}^{\prime}\right) \cong\left(S_{L},\left[s_{x} \mid s_{y}\right], S_{R}\right)$.

Now, consider the bipartition $\left(S_{L},\left[s_{x} \mid S_{y}\right], S_{R}\right)$. Since we are interested only in a sufficient condition, we can safely do the replacement $s_{x} \mapsto s_{1}$ obtaining the bipartition $\left(S_{L}^{\prime},\left[s_{1} \mid s_{y}\right], S_{R}\right)$ with $S_{L}^{\prime}=\left\{s_{1}, \ldots, s_{j}\right\} \backslash\left\{s_{1}\right\}$.

It is thus immediate that $\left(S_{L},\left[s_{x} \mid s_{y}\right], S_{R}\right) \cong\left(S_{L}^{\prime},\left[s_{1} \mid s_{y}\right]\right.$, $\left.S_{R}\right)$. Next, recalling that the measurements are local, we can combine $S_{L}^{\prime}$ with $S_{R}$ and write $\left(S_{L}^{\prime},\left[s_{1} \mid s_{y}\right], S_{R}\right) \cong$ $\left(\left[s_{1} \mid s_{y}\right], S_{L}^{\prime} \cup S_{R}\right), \quad$ hence $\quad\left(S_{L},\left[s_{x} \mid s_{y}\right], S_{R}\right) \cong\left(\left[s_{1} \mid s_{y}\right]\right.$, $\left.S_{L}^{\prime} \cup S_{R}\right)$.

It follows from the above argument that bipartitions $\left\{\left(\left[s_{1} \mid s_{y}\right], S_{R}\right): \forall s_{y} \in\left\{s_{2}, \ldots, s_{n}\right\}\right\}$ cover the set of all possible bipartitions, in the sense that detection of their conditional entanglement is sufficient to certify entanglement in all possible bipartitions, and hence GME. Since this set has $n-1$ elements corresponding to the $n-1$ pairings of $s_{1}$ with other subsystems, the detection of GME requires only a linear number of bipartitions.

It is worth mentioning that, despite the systematic reduction in the number of bipartitions needed for the certification of GME, in general, there exist GME states the entanglement of which cannot be detected through localizing procedure as described in Theorem 1. In particular, it was shown by Mičuda et al. [60] that there exist three-qubit mixed states that are genuinely multipartite entangled and, yet, no measurement on any of the subsystems leads to a bipartite entangled state of the remaining subsystems. An example state with this property is the Werner state [47]

$$
\varrho_{W}=\frac{1-p}{8} I+p\left|\mathrm{GHZ}_{+}^{\otimes 3}\right\rangle\left\langle\mathrm{GHZ}_{+}^{\otimes 3}\right|
$$

for $1 / 5<p \leq 1 / 3$. Moreover, very recently, a related result within the context of device-independent GME certification was obtained independently by Zwerger et al. [61]. There, it was shown that all pure GME states contain conditional bipartite entanglement. As a corollary, one can say that if a pure state is GME, then its GME can necessarily be certified using our conditional GME witnessing technique. Interestingly, it is possible to reformulate Theorem 1 in terms of neighboring subsystems of an $n$-partite system.

Theorem 2. Consider an n-partite quantum system of any dimension. Then, the certification of entanglement in all conditional bipartitions $\left[s_{x} \mid s_{x+1(\bmod n)}\right]$, with $1 \leq x \leq n$, conditioned on an outcome $i$ of suitable local measurements on the remaining $n-2$ subsystems is sufficient for

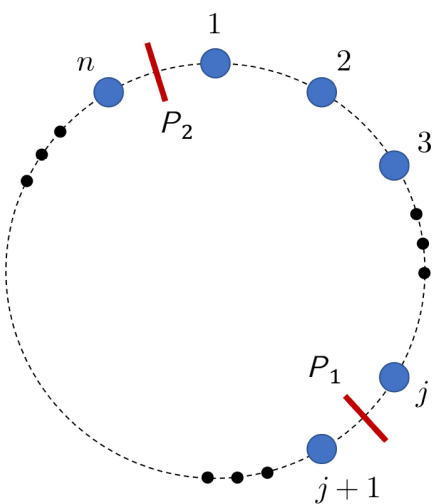

FIG. 5. Schematic of the bipartition $\left(s_{1}, \ldots, s_{j} \mid s_{j+1}, \ldots, s_{n}\right)$ in neighboring subsystems for $n$ qubits arranged on a circle. The placements of separators $P_{1}$ and $P_{2}$ suffice to specify any given bipartition of the system.

the certification of GME of the system. The number of bipartitions needed to be checked is thus $n-1$.

Proof. It is possible to give an intuitive pictorial proof of this theorem. Suppose that, as shown in Fig. 5, the $n$ subsystems are arranged on a circle so that subsystems $s_{1}$ and $s_{n}$ are neighbors. A given bipartition $\left(s_{1}, \ldots, s_{j} \mid s_{j+1} \ldots, s_{n}\right)$ can be identified with the placements of two separators $P_{1}$ and $P_{2}$. Now, suppose that $P_{1}$ is placed between two subsystems $s_{j}$ and $s_{j+1}$ and suppose that, conditioned on local measurements on the remaining subsystems, we find that $s_{j}$ and $s_{j+1}$ are entangled. It is immediate, using Lemma 1, that due to the locality of measurements the placement of the separator $\mathrm{P}_{2}$ is irrelevant, so that a nonzero LE between $s_{j}$ and $s_{j+1}$ implies entanglement within all bipartitions that can be obtained by displacing separator $P_{2}$.

Finally, since the placements of $P_{1}$ and $P_{2}$ are sufficient to specify all bipartitions, we conclude that the only relevant degree of freedom is the placement of $P_{1}$. Finally, the latter can be done in $n-1$ ways, hence the result.

Lastly, let us note that in Theorems 1 and 2 we focus on local measurements. However, it is possible to derive variants of them in which joint entangled measurements on specific subsystems are allowed. Due to the fact that joint measurements generally increase the localization power, it is expected to obtain more powerful but rather complicated entanglement criteria from such considerations.

\section{Conditional entanglement witnessing}

Given an $n$-partite system, there are many ways to verify the entanglement localized between two subsystems by measuring the rest, and then using either Theorem 1 or 2 to show GME. Additionally, we require the detection of 
each nonzero LE to be efficient for each bipartition. A particular approach to do the latter is witnessing $[62,63]$. The collection of all quantum states with unlocalizable entanglement with respect to a specific conditional bipartition $\left[s_{x} \mid s_{y}\right]$ form a closed convex set denoted by $\mathcal{S e p} p_{\left[s_{x} \mid s_{y}\right]}$. It is worth emphasizing that the states in $\mathcal{S} e p_{\left[s_{x} \mid s_{y}\right]}$ are not necessarily biseparable states, rather they are states that reduce to separable bipartite states upon any local measurements on $\left\{s_{x}, s_{y}\right\}^{\mathrm{c}}$. It thus follows that $\mathcal{S e p}_{\left[s_{x} \mid s_{y}\right]} \supset \mathcal{S e p}_{\mathbf{I}_{1} \mid \mathbf{I}_{2}}$ for any $\mathbf{I}_{1} \ni s_{x}$ and any $\mathbf{I}_{2} \ni s_{y}$. For every $n$-partite state $\varrho \notin$ $\mathcal{S e p}_{\left[s_{x} \mid s_{y}\right]}$, that is a state with LE with respect to the conditional bipartition $\left[s_{x} \mid s_{y}\right]$, there exists a bounded Hermitian operator $W_{\left[s_{x} \mid s_{y}\right]}$ of the form

$$
W_{\left[s_{x} \mid s_{y}\right]}=W_{s_{x} \mid s_{y}} \bigotimes_{z \in\left\{s_{x}, s_{y}\right\}^{\mathrm{c}}} M_{s_{z}: i}
$$

with $M_{s_{z}: i}$ being the effect corresponding to the outcome $i$ of some specific measurement on the subsystem $s_{z}$, such that

$$
\begin{aligned}
\forall \sigma \in \mathcal{S e p}_{\left[s_{x} \mid s_{y}\right]} \operatorname{Tr}\left(W_{\left[s_{x} \mid s_{y}\right]} \sigma\right) \geq 0, \\
\operatorname{Tr}\left(W_{\left.\left[s_{x} \mid s_{y}\right] \varrho\right)}<0 .\right.
\end{aligned}
$$

We call the operator $W_{\left[s_{x} \mid s_{y}\right]}$ a conditional entanglement witness, the existence of which is guaranteed by (i) the assumption that $\varrho$ contains LE between $s_{x}$ and $s_{y}$, namely $\varrho \notin \mathcal{S} e p_{\left[s_{x} \mid s_{y}\right]}$, and (ii) as described in Sec. IV A, by the Hahn-Banach separation theorem, there exists a witness $W_{s_{x} \mid s_{y}}$ for the entanglement concentrated between these two subsystems. Furthermore, for each pair $s_{x}$ and $s_{y}$, the bipartite witness $W_{s_{x} \mid s_{y}}$ can be constructed from a test operator $L_{s_{x} \mid s_{y}}$ using the approach delineated in Sec. IV A. It is worth emphasizing that, determining the separability bound for the test operator $L_{s_{x} \mid s_{y}}$ to be used in the construction of $W_{s_{x} \mid s_{y}}$ in Eq. (24) requires an optimization over biseparable states of systems $s_{x}$ and $s_{y}$. Hence, the complexity of determining this bound is the same as generic entanglement witnessing, i.e., it is efficiently decidable $[53,54]$. Combining conditional entanglement witnessing with either of Theorems 1 and 2 thus gives an efficient technique for verification of GME that maintains the practicality of the entire process with additional robustness. We call this approach conditional GME witnessing.

In the rest of this paper, we apply the conditional GME witnessing to QEC stabilizer measurement circuits to characterize their performance in terms of their power for the creation of GME states in the presence of errors, different sources of noise and inefficiencies. We show that with a linear (in the number of qubits) increase in the complexity of the approach, we obtain significant noise robustness in our technique compared to the standard single-witness entanglement tests [29]. Our claim can be pictorially understood as shown in Fig. 4. Any standard GME witness can detect only GME states that do not belong to the closed convex hull of sets of separable states with respect to each bipartition. There, however, exist GME states that belong to this convex hull. Using conditional GME witnessing, in general, we use a linear number of witnesses to detect such GME states and obtain a higher resolution in the detection of GME.

\section{NOISY TRAPPED-ION CIRCUITS}

Starting with the pioneering proposal of Cirac and Zoller for universal quantum computation using the internal states of trapped ions as qubits, and the vibrational modes as a quantum bus to mediate entangling gate operations $[64,65]$, systems of trapped atomic ions in radio-frequency potentials are nowadays considered to be among the most promising quantum-information processors $[1,66]$. Over the years, various quantum protocols have been realized in different trapped-ion platforms [66,67], including smallscale QEC algorithms [68,69], a topologically encoded qubit based on the seven-qubit color code [16], faulttolerant error detection [14], deterministic correction of qubit loss [70], and the first entangling gate at the level of logical qubits [71]. One of the current quests in trapped-ion quantum computing is to scale up these prototype processors towards larger-scale systems capable of taking full advantage of QEC routines of FT [4,15,45,72-74].

\section{A. Compilation into native ion-trap gates and operations}

Whilst a large-scale FT implementation of QEC for universal quantum computations is beyond the reach of nearterm devices, small-scale FTQEC protocols can already be run on some of the current trapped-ion platforms. Even though they still consist of a reduced number of qubits where some decoherence is unavoidable, they deliver long coherence times $[75,76]$ and high-fidelity single and twoqubit gates [77-80] that are essential to test the performance of small-distance FTQEC codes or its building blocks. We thus focus on this platform to demonstrate the robustness and efficiency of our entanglement characterization method.

The native trapped-ion entangling gates in current designs are not CNOT gates [8]. Rather, they are based on state-dependent dipole forces and effective spin-spin interactions [81-84]. In this work, we are concerned with the so-called $Z$-state-dependent forces, which give rise to the entangling $Z Z$ gates $[78,79,84]$, namely

$$
U_{i j}^{Z Z}(\theta)=e^{-\mathrm{i} \frac{\theta}{2} Z_{i} Z_{j}}
$$

in which $Z_{i}$ and $Z_{j}$ are the Pauli matrices of the corresponding $i$ th and $j$ th qubits involved in the gate, respectively, and $\theta$ is the corresponding pulse area. In addition to $Z Z$ gates, which are fully entangling for $\theta=\pi / 2$, i.e., they 


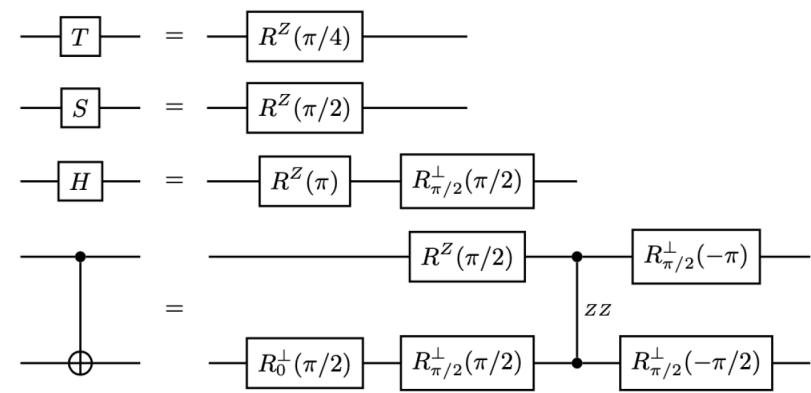

FIG. 6. Correspondence between the standard and trapped-ion universal gate sets enabling quantum computation. The solid line that joins two filled circles represents the $Z Z$ gate $U_{i j}^{Z Z}(\pi / 2)$ in Eq. (26).

map product states onto GHZ-type entangled states, we also consider single-qubit rotations. In particular, rotations of the form

$$
R_{i}^{Z}(\theta)=e^{-\mathrm{i} \frac{\theta}{2} Z_{i}}
$$

are obtained by local ac-Stark shifts [85]. The parallel rotations of ion states within the equatorial plane of the Bloch sphere are obtained by a simultaneous driving of the carrier transition of the ions in the laser focus,

$$
R_{\phi, i}^{\perp}(\theta)=e^{-\mathrm{i} \frac{\theta}{2} \sum_{i}\left(\cos \phi X_{i}+\sin \phi Y_{i}\right)},
$$

where $R_{0, i}^{\perp}(\theta)$ and $R_{\pi / 2, i}^{\perp}(\theta)$ correspond to rotations around $x$ and $y$ axes of the Bloch sphere, respectively. For instance, by setting $\phi=0$ and $\theta=\pi$, Eq. (28) represents single-qubit $\pi$ pulses applied to all illuminated ions. We note that, although these rotations act on all illuminated ions, the equatorial rotations can be applied to a particular set of ions by using spin-echo-type refocusing pulses that interleave these rotations with the addressable $Z$-type rotations [86].

Although this collection of gates is not the standard one in quantum computation [8], it is a universal gate set so that any quantum algorithm can be decomposed into a sequence of these elementary operations [86,87]. In Fig. 6, we show the correspondence between the standard universal gate set and certain sequences of native trapped-ion gates. In Fig. 7, on the other hand, we present the compiled circuit of Fig. 2(a) into the native trapped-ion gate set.

The entangling $Z Z$ gates for $\theta=\pi / 2$ between the $i$ th and $j$ th qubit can be written as $U_{i, j}^{Z Z}(\pi / 2)=(\mathbb{I}-$ $\left.\mathrm{i} Z_{i} Z_{j}\right) / \sqrt{2}$. Similarly one can write the single-qubit rotations for qubit $i$ th as $R_{\pi / 2, i}^{\perp}( \pm \pi / 2)=\left(\mathbb{I} \mp \mathrm{i} Y_{i}\right) / \sqrt{2}$ and

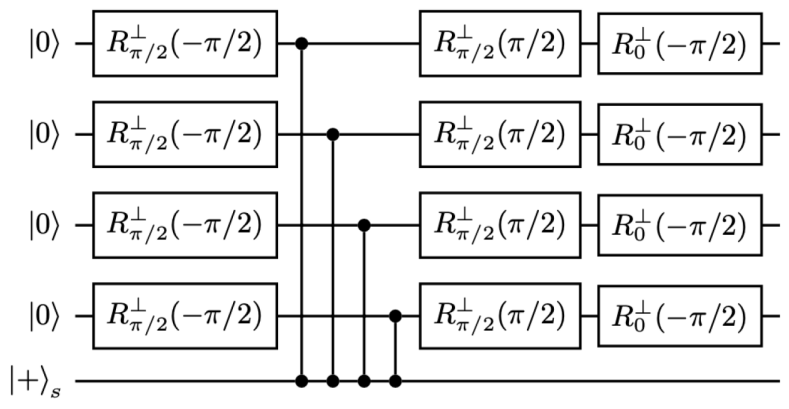

FIG. 7. Non-FT circuit using the native trapped-ion gates.

$R_{0, i}^{\perp}(-\pi / 2)=\left(\mathbb{I}+\mathrm{i} X_{i}\right) / \sqrt{2}$. After some algebra, the output state of the circuit in Fig. 7 is obtained as

$$
\left|\psi_{\text {out }}\right\rangle=\frac{|0\rangle^{\otimes 5}+|1\rangle^{\otimes 5}}{\sqrt{2}}=\left|\mathrm{GHZ}_{+}^{\otimes 5}\right\rangle
$$

which, as expected, is identical to the five-qubit GHZ state in Eq. (4) generated with the circuit comprising CNOT gates. Similarly to the non-FT scenario with CNOT gates, we may analyze the propagation of a dangerous single $X$ error from the syndrome qubit to the data qubits through the $Z Z$ gates, the details of which are given in Appendix D. It follows that, in this case too, the syndrome readout will not reveal any information about the propagation of the error. This leads us to the flag-based circuit shown in Fig. 8, which is the compiled version of Fig. 2(b) into the native trapped-ion gate set. It is straightforward to calculate the output of this circuit as

$$
\left|\psi_{\text {out }}\right\rangle=\frac{\left(|0\rangle^{\otimes 5}+|1\rangle^{\otimes 5}\right)|+\rangle_{f}}{\sqrt{2}}=\left|\mathrm{GHZ}_{+}^{\otimes 5}\right\rangle|+\rangle_{f} .
$$

One realizes that the sequential application of two $Z Z$ gates between the syndrome qubit $s$ and the flag qubit $f$, $U_{s f}^{Z Z}(\pi / 2) U_{s f}^{Z Z}(\pi / 2)|-\rangle_{s}|-\rangle_{f}$, leaves the two qubits in the product state $|+\rangle_{s}|+\rangle_{f}$. A dangerous Pauli $X$ error in the syndrome that produces a cascade of errors in the data, however, will flip the flag back into $|-\rangle_{f}$. Hence, a readout of the qubit $f$ "flags" the propagation of multiple errors that would not be possible in a non-FT scheme.

In parallel with Fig. 3(d), whenever the syndrome qubit suffers from a coherent-rotation error $\exp (i \phi X)$ between the second and third syndrome-data $Z Z$ gates, as shown in Fig. 9, the ideal output state can be easily computed as

$$
\begin{aligned}
\left|\psi_{\text {out }}\right\rangle= & \cos \phi|+\rangle_{f}\left|\mathrm{GHZ}_{+}^{\otimes 5}\right\rangle \\
& +\mathrm{i} \sin \phi|-\rangle_{f} Y_{s} X_{3} X_{4}\left|\mathrm{GHZ}_{+}^{\otimes 5}\right\rangle .
\end{aligned}
$$

A projective measurement of the flag again unveils a cascaded error in the syndrome and data qubits. A similar procedure can be implemented using the $g_{z}$ generator as presented in Appendix C. 


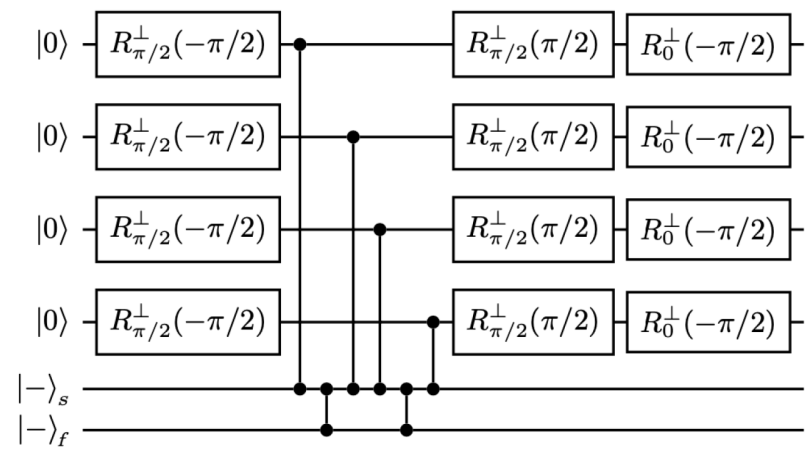

FIG. 8. Flag-based FT circuit using the native trapped-ion gates.

\section{B. Noise models}

Recall from Sec. IV A that, in general, the output of an ideal plaquette is a GME state. Hence, to assess the robustness of the conditional GME witnessing method in a trapped-ion platform, we implement different noise models into the circuit, and compare the performance of conditional GME witnessing against the standard singlewitness (i.e., fidelity measurement) approach in detecting the potential GME output state.

In this regard, we use two different circuit-noise models and one measurement error scheme as leading errors in the stabilizer circuits for each of the non-FT and flagbased FT scenarios. As the first circuit noise model we use a simplified and phenomenological one in which the preparation of the input state and all the gates are assumed to be perfect while the decoherence of qubits is simulated by an independent single-qubit depolarizing channel [8] applied to each qubit right before their measurements. Our second noise model is motivated by the realistic experimental details discussed in Ref. [79] wherein shuttling techniques are employed to carry out the two-qubit gates. Thus, we consider the case wherein the preparation of the input state is perfect while the two-qubit gates in the circuit suffer from independent depolarizing noise.

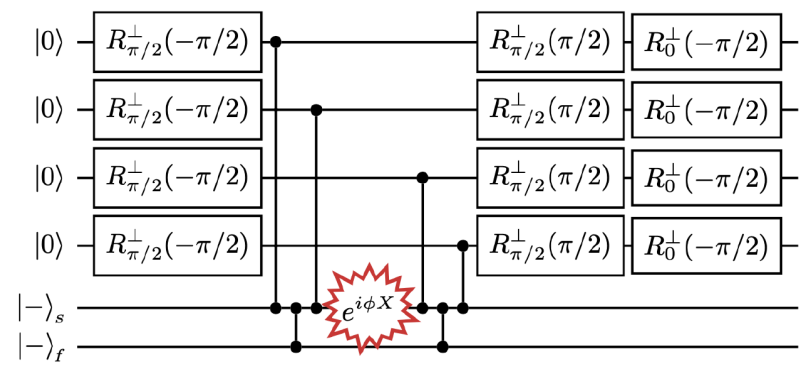

FIG. 9. Flag-based FT stabilizer measurement circuit using the native trapped-ion gates. Here, a coherent-rotation error $e^{i \phi X}$ is injected in a specific position to create a six-qubit entangled state in the flag-based FT stabilizer measurement.
The measurement errors, modeled as independent classical bit-flip errors in the measured bits, are present in both scenarios and for all qubits. We assume that dephasing effects in idle qubits are depreciated compared to the two-qubit errors in entangling gates. This is a realistic assumption given the recent experiments on the use of stable magnetic fields, which lead to a negligible dephasing of the qubits during the idle-time intervals [88]. Moreover, in the considered experimental layout [79], single-qubit gates also have a negligible error in comparison to the two-qubit and measurement errors, and can be thus neglected. We do assume here that the error rates per two-qubit gate increase with the depth of the circuit as a consequence of the shuttling-based approach used in quantum charge-coupled devices [89-91].

\section{Independent depolarizing noise}

This error channel is used only for the phenomenological model. It consists of independent depolarizing channels of the form

$$
\epsilon_{i}^{\mathrm{dip}}(\varrho)=(1-p) \varrho+\frac{p}{3} \sum_{\sigma \in\{X, Y, Z\}} \sigma_{i} \varrho \sigma_{i},
$$

acting on each of the qubits at the end of the circuit sequence and just before the measurements. Here, $p$ is probability for a depolarizing error to occur on the $i$ th qubit and we consider it to be the same for all qubits.

\section{Two-qubit depolarizing noise}

In a trapped-ion platform, ions can be shuttled in and out of the laser interaction zone in which the quantum operations and readouts are carried out [79]. This process may excite the ions' vibrational modes that affect the fidelity of the two-qubit gates depending on their order in the shuttling sequence or, equivalently, the time step at which the gate is applied. This detrimental effect can be modeled in a conservative manner by a two-qubit depolarizing noise after the application of each two-qubit entangling gate $U_{i j}^{Z Z}$.

For each pair of ions involved in a $Z Z$ gate, they may undergo 15 possible one- and two-qubit Pauli errors so that the total error channel is described as

$$
\epsilon_{i, j}^{\mathrm{dip}}(\varrho)=[1-p(r, t)] \varrho+\frac{p(r, t)}{15} \sum_{\sigma, \kappa \in\{I, X, Y, Z\}} \sigma_{i} \kappa_{j} \varrho \kappa_{j} \sigma_{i},
$$

where $i$ and $j$ with $i \neq j$ denote the active ions involved in each two-qubit entangling gate, $p$ is the error probability, and the sum on the right runs over the six nontrivial singlequbit and the nine nontrivial two-qubit Pauli operators. We assume a time- (or circuit depth-) dependent exponential growth in the error probability defined as $p(r, t)=$ $p(1+r)^{t}$, where $r$ and $t$ are the error-growth rate per gate 
and the time step at which $Z Z$-entangling gates are applied, respectively. It is worth to clarify that $t$ is not the actual elapsed physical time in an experimental implementation rather it is an index taking values $t \in \mathbb{N}$ specifying the order of entangling gates in the circuit. Hence, we assume the time increment between every two entangling gate to be $\Delta t=1$. Upon using this model, we also consider three error-growth rates of $r=0, r=0.1$, and $r=0.2$, noting that an $r=0.2$ implies that the fourth and sixth two-qubit gates are about 2 and 3 times worse than the first one, respectively.

\section{Bit-flip measurement errors}

Entanglement witnessing procedures with and without conditioning involve Pauli measurements of both data and ancillary qubits, which may also suffer from imperfections. We use a simple model for measurement errors, namely independent bit flips. In a measurement of a singlequbit Pauli operator $\sigma \in\{X, Y, Z\}$ the corresponding errorfree positive operator-valued measure $(\mathrm{POVM})[8]$ is specified by the set of operators $\left\{E_{+}^{\sigma}=(I+\sigma) / 2, E_{-}^{\sigma}=\right.$ $(I-\sigma) / 2\}$. For each measurement outcome, a bit-flip error taking place with probability $p_{\mathrm{ME}}$ thus gives rise to the POVM effects

$$
\begin{aligned}
e_{+}^{\sigma} & =\left(1-p_{\mathrm{ME}}\right) E_{+}^{\sigma}+p_{\mathrm{ME}} \kappa^{\sigma} E_{+}^{\sigma} \kappa^{\sigma}, \\
& =\left(1-p_{\mathrm{ME}}\right) E_{+}^{\sigma}+p_{\mathrm{ME}} E_{-}^{\sigma},
\end{aligned}
$$

and

$$
\begin{aligned}
e_{-}^{\sigma} & =\left(1-p_{\mathrm{ME}}\right) E_{-}^{\sigma}+p_{\mathrm{ME}} \kappa^{\sigma} E_{-}^{\sigma} \kappa^{\sigma}, \\
& =\left(1-p_{\mathrm{ME}}\right) E_{-}^{\sigma}+p_{\mathrm{ME}} E_{+}^{\sigma} .
\end{aligned}
$$

Here, $\kappa^{\sigma} \in\{X, Y, Z\}$ is the (measurement-dependent) error operator such that $\kappa^{\sigma} E_{+}^{\sigma} \kappa^{\sigma}=E_{-}^{\sigma}$ and $\kappa^{\sigma} E_{-}^{\sigma} \kappa^{\sigma}=E_{+}^{\sigma}$. It is also obvious that the effect of measurement errors can instead be described by a quantum channel acting on the individual qubits such that

$$
\epsilon_{i}^{\sigma}(\varrho)=\left(1-p_{\mathrm{ME}}\right) \varrho+p_{\mathrm{ME}} \kappa^{\sigma} \varrho \kappa^{\sigma} .
$$

\section{ROBUSTNESS OF CONDITIONAL GME WITNESSING}

The experimental implementation of any entanglement witnessing technique - in fact, any experimental characterization approach relying on surpassing some threshold value - comes with experimental error bars resulting from standard statistical uncertainty due to a finite number of measurements and possibly systematic errors from experimental miscalibrations. It is the standard practice to call the witnessing inconclusive when the separability bound lies within the confidence region of the experimental estimation. Refuting this problem is possible in two ways: by reducing statistical errors with larger sets of data and improving the quality of the measurement elements, or by using a more robust witnessing method to amplify the violation of the threshold such that, for a fixed confidence interval associated to the measured data, one obtains a reliable witness value.

We are now ready to examine the robustness and efficiency of our conditional GME witnessing technique compared to the standard GME certification approaches used within the literature. To this end, we consider noisy nonFT and flag-based FT plaquette measurement circuits and witness the resulting entangled state using imperfect measurements in three different ways: (i) standard witnessing with a GHZ projector (i.e., a single witness) requiring an exponential number of measurements, that is, by measuring fidelity to the ideal output state, (ii) standard-linear witnessing (SL) with a single witness that relies on only a linear number of measurements using the proposed witnesses of Tóth and Gühne [29], and (iii) our proposed conditional GME witnessing requiring a linear number of witnesses and measurements.

\section{A. Conditional GME witnessing in the noisy non-FT plaquette circuit}

Consider the five-qubit non-FT $g_{x}$ circuit from Fig. 7. We implement each noise model of Sec. VB on the circuit and witness the output entangled state using the three above-mentioned techniques. Beginning with the standard witnessing, it is shown in Sec. IV A [cf. Eq. (18)] that the five-qubit GHZ projector is a possible test operator for GME,

$$
L_{\mathrm{GHZ}} \otimes 5=\left|\mathrm{GHZ}_{+}^{\otimes 5}\right\rangle\left\langle\mathrm{GHZ}_{+}^{\otimes 5}\right| .
$$

It is also shown in Eq. (20) that the detection bound for this test operator is $1 / 2$ for any bipartition, i.e., given any state $\varrho$, an expectation value satisfying $\operatorname{Tr}\left(\varrho L_{\mathrm{GHZ}} \otimes 5\right)>1 / 2$ implies the entanglement of $\varrho$ with respect to all bipartitions and thus its GME. We also show in Eq. (6) that the five-qubit GHZ projector can be written in terms of its $2^{5}$ stabilizers. Combining the two, we observe that the expectation value of $L_{\mathrm{GHZ}} \otimes 5$ for any state is given by

$$
\left\langle L_{\mathrm{GHZ}}{ }^{\otimes 5}\right\rangle=\operatorname{Tr}\left(\varrho L_{\mathrm{GHZ}}{ }^{\otimes 5}\right)=\frac{1}{2^{5}} \sum_{i=1}^{2^{5}}\left\langle S_{i}\right\rangle .
$$

It is also worth pointing out that $\left\langle L_{\mathrm{GHZ}}{ }^{\otimes 5}\right\rangle$ equates to the fidelity of the state $\rho$ with the ideal output state of the fivequbit non-FT plaquette circuit derived in Eq. (4). For the standard witnessing of GME using the five-qubit GHZ projector $L_{\mathrm{GHZ}}{ }^{\otimes 5}$, it is thus sufficient to locally measure the stabilizers at the output of the plaquette circuit the number of which would in general scale exponentially with the size of the register. 
The second approach we consider is the SL witnessing of GME using a witness constructed out of the five generators of the five-qubit stabilizer subgroup given in Eq. (5). Two test operators introduced by Tóth and Gühne [29] for this purpose are, after normalization,

$$
L_{\mathrm{TG} 1}=\frac{1}{2} \frac{I+g_{5}}{2}+\frac{1}{2} \prod_{i=1}^{4} \frac{I+g_{i}}{2}
$$

and

$$
L_{\mathrm{TG} 2}=\frac{1}{5} \sum_{i=1}^{5} g_{i}
$$

The GME bounds for these witnesses are $l_{\mathrm{TG} 1}=3 / 4$ and $l_{\mathrm{TG} 2}=4 / 5$, respectively [29]. Noting the form of the stabilizer generators, the obvious advantage of these witnesses is that they require only two measurement settings.

Finally, we implement our proposed conditional GME witness and show through this example that it is technically simple and, at the same time, powerful. Using Theorem 1, we focus merely on the four conditional bipartitions $[s \mid 1]$, $[s \mid 2],[s \mid 3]$, and $[s \mid 4]$ and introduce the conditional test operators

$$
\left.L_{[s \mid x]}=\mid \text { Bell }\right\rangle_{s \mid x}\langle\text { Bell }|\otimes|+\rangle\left\langle+\left.\right|^{\otimes 3}\right.
$$

for $x \in\{1,2,3,4\}$. Here, $\mid$ Bell $\rangle_{s \mid x}\langle$ Bell $|$ is the projection of the qubit pair $s$ and $x$ onto the Bell state $\mid$ Bell $\rangle=$ $(|00\rangle+|11\rangle) / \sqrt{2}$ and $|+\rangle+\left.\right|^{\otimes 3}$ denotes the projector of the remaining three qubits $\{s, x\}^{\mathrm{c}}$. The bounds on the conditional test operators are easy to compute as we are effectively dealing with two-qubit systems. For the case of interest here, it is known that

$$
l_{s \mid x}=\sup _{\sigma \in \mathcal{S} e p_{s \mid x}} \operatorname{Tr}\left(\sigma|\operatorname{Bell}\rangle_{s \mid x}\langle\operatorname{Bell}|\right)=\frac{1}{2},
$$

where the supremum is taken over the set of all bipartite separable states of qubits $s$ and $x$. Consequently, for any conditional bipartition $[s \mid x]$ with $x \in\{1,2,3,4\}$ and any given five-qubit state $\varrho$, an expectation value $\left\langle L_{[s \mid x]}\right\rangle>1 / 2$ for all $x \in\{1,2,3,4\}$ implies GME of the quantum state $\varrho$. Importantly for us, the Bell state is the one-dimensional code space of the stabilizer generators $\{X X, Z Z\}$. Similar to Eq. (6) we thus have

$$
|\mathrm{Bell}\rangle_{s \mid x}\langle\mathrm{Bell}|=\frac{I_{s} I_{x}+X_{s} X_{x}-Y_{s} Y_{x}+Z_{s} Z_{x}}{4},
$$

implying that conditional GME witnessing can be performed solely by local Pauli measurements on the qubits. Furthermore, for the implementation of conditional GME witnessing using $L_{[s \mid x]}$ of Eq. (41) we need three settings per bipartition, hence a total of 12 measurement settings. It is also noteworthy that, while we consider only the projection onto $|+\rangle\left\langle+\left.\right|^{\otimes 3}\right.$, we can consider other outcome combinations of the $X$ measurements on these three qubits that give rise to either the same Bell state or the one orthogonal to it, namely $\left(|00\rangle_{s \mid x}-|11\rangle_{s \mid x}\right) / \sqrt{2}$, that can be witnessed seamlessly with the same measurement settings. In experimental implementations of the conditional GME witnessing, in general, more than one of the conditioning events can thus be used to keep the overall witnessing procedure efficient costing at most a linear overhead in the number of settings.

We now turn to evaluating the robustness of our technique versus other witnessing approaches in the literature.

\section{Phenomenological depolarizing noise model}

The contour plots of Fig. 10 present our results for the four witnessing techniques applied to the five-qubit nonFT plaquette readout circuit (see Fig. 7) subjected to the noise channel of Eq. (32) and the measurement noise of Eq. (36). From left to right, the four subfigures represent the results obtained for (a) the standard witness of Eq. (37), (b) the SL witness of Eq. (39), (c) the SL witness of Eq. (40), and (d) our conditional witness of Eq. (41) for $x=1$. The dark blue shaded areas in all plots represent states in which their GME, if any, cannot be detected by the corresponding witness. One can thus clearly appreciate the significant increase in the noise tolerance of conditional GME witnessing compared to the other witnessing methods that can be found in the previous literature.

In particular, for $p_{\mathrm{ME}}=0$, the highest depolarizing error probability $p$ that can be afforded before losing the GME witnessing capabilities for the standard method [Fig. 10(a)] is $p \approx 0.13$. For the first [Fig. 10(b)] and the second [Fig. 10(c)] SL witness we find $p \approx 0.09$ and $p \approx 0.07$, respectively. These values demonstrate the typical trade-off between the number of measurements and the robustness of witnesses. For our conditional GME witness [Fig. 10(d)], however, the error tolerance goes up to $p \approx 0.2$. We thus observe the counterintuitive fact that a linear number of measurements does not necessary imply a loss of robustness in GME detection. Similarly, by fixing $p=0$ for the depolarizing noise, we notice that the measurement probability error $p_{\mathrm{ME}}$ also reaches its highest threshold value for our conditional witnessing with $p_{\mathrm{ME}} \approx$ 0.2 compared to $p_{\mathrm{ME}} \approx 0.1$ for the standard method. We see the lowest robustness for the first and the second SL witness with $p_{\mathrm{ME}} \approx 0.07$ and $p_{\mathrm{ME}} \approx 0.05$, respectively.

Recall that, in the conditional GME witnessing method for the five-qubit circuit, according to Theorem 1, it is needed to test entanglement in a total of four bipartitions. In the top panel of Fig. 11, we present the behavior of the expectation value $\left\langle L_{[s \mid x]}\right\rangle$ with respect to the depolarizing error probability $p$ for different $x \mathrm{~s}$ at the fixed measurement 

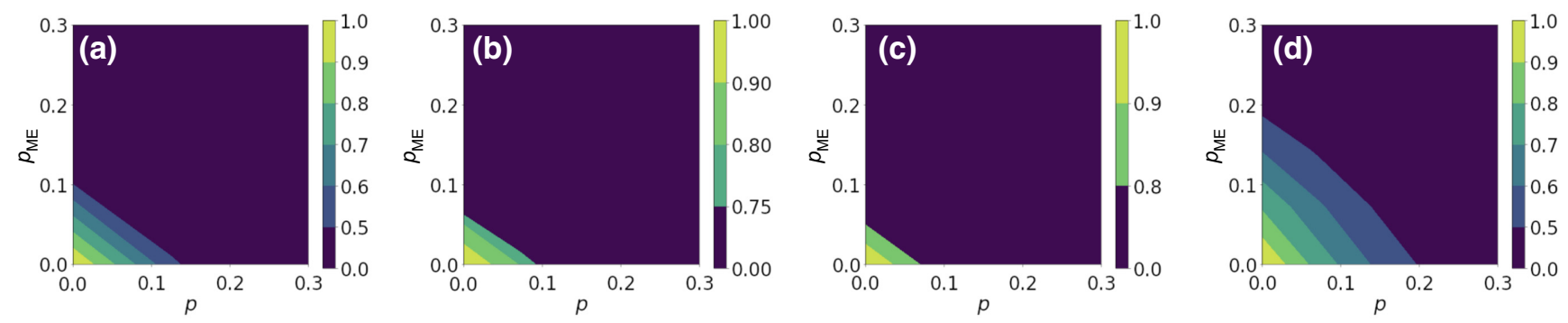

FIG. 10. GME witnessing plots in a five-qubit non-FT plaquette readout circuit under the phenomenological depolarizing noise model. Each contour plot represents, for different values of depolarizing-noise probability $p$ and measurement-error probability $p_{\mathrm{ME}}$, the expectation values of (a) the standard GME witness $L_{\mathrm{GHZ}}{ }_{55}$ of Eq. (38), (b) the first SL witness, $L_{\mathrm{TG} 1}$ in Eq. (39), (c) the second SL witness $L_{\mathrm{TG} 2}$ in Eq. (40), and (d) the worst-case conditional witnessing using $L_{[s \mid x]}$ in Eq. (41) for $x=1$. The colormap sidebars represent the witness bounds for each method. In all cases, the dark blue region identifies the noise values for which the GME tests are inconclusive.

error probability $p_{\mathrm{ME}}=0$. It is evident that for this noise model the four bipartitions behave identically, as expected from the symmetries of the ideal state, the noise model exploited, and the conditional GME test used. Therefore, in this case, all the bipartitions would exhibit the same threshold values as in Fig. 10(d).

\section{Two-qubit depolarizing noise model}

As mentioned earlier, the depolarizing noise model of Sec. VB is an oversimplification. Hence, in this section, we assume a five-qubit non-FT readout circuit for the plaquette that suffers from the more realistic circuit noise of Eq. (33). As discussed in Sec. VB, this error model introduces a two-qubit depolarizing channel after each entangling gate. Recall that, in this model, the two-qubit gate error probability $p(r, t)$ grows exponentially with the gate time step $t$ at which $Z Z$-entangling gates are applied at the rate $r$. Hence, the model takes into account the fact that the fidelity of gates can be affected by the depth of the circuit due to the accumulated decoherence and noise effects. As before, we also include the measurement noise effects as per Eq. (36).

Figure 12 represents the resulting contour plots of the expectation values of (a) the standard witness of Eq. (37), (b) the SL witness of Eq. (39), (c) the SL witness of Eq. (40), and (d) our conditional witness of Eq. (41) for $x=1$. Our choice of the conditional bipartition in Fig. 12(d) is due to the fact that, here, in contrast to the phenomenological noise, the expectation values $\left\langle L_{[s \mid x]}\right\rangle$ are not equal for $x=1,2,3,4$, as shown in the lower panel of Fig. 11. In this case, the conditional bipartition $[s \mid 1]$ is the one in which the conditional GME witnessing performs the worst, providing thus the most stringent conditions for the detection of GME. Notably, the latter counterintuitive observation suggests that the depolarizing noise processes before a two-qubit gate are more detrimental than those taking place after it to the correlation created by that gate between the target qubit and all the previously entangled qubits. This could be due to the loss of the input coherence that is necessary for correlating the qubits [92]. As such, qubit 3 , for instance, becomes less correlated with qubits $(s, 1,2)$ than qubit 2 with qubits $(s, 1)$, meaning that conditioning on qubit 3 is less informative than conditioning on qubit 2 . Hence, the conditional bipartition $[s \mid 3]$ conditioned on the more informative qubits 1,2 and the less informative qubit 4 performs better compared to the conditional bipartition $[s \mid 2]$ conditioned on the more informative qubits 1 and the less informative qubits 3,4 .

In Fig. 12 the dark blue shaded area again indicates the states for which each of the witnessing techniques is inconclusive [93]. We observe that with increasing error-growth rate $r$ the threshold for $p$ decreases. However, even in the worst-case scenario, our conditional GME witnessing represented in Fig. 12(d) shows significant robustness compared to the previously studied entanglement witnesses for each $r$.

It is also interesting to compare the effect of the two different noise models shown in Figs. 10 and 12. We notice a higher threshold of detectable GME states when the twoqubit depolarizing noise model is applied. This means that the phenomenological model may underestimate the performance of entanglement witnessing methods whilst the more realistic two-qubit depolarizing noise model predicts a higher robustness of our conditional GME detection. Nevertheless, it is evident from our analysis here that for both noise models the GME witnessing by conditioning method introduced in this text is not only efficient in the number of qubits, but also robust against noise.

\section{B. Conditional GME witnessing in noisy flag-based FT plaquette circuit}

We now consider the flag-based FT readout circuit for a single plaquette and implement each noise model of Sec. VB on the circuit evaluating the different GME witnesses on the output entangled state. We use again the three mentioned techniques: (i) standard witnessing with 

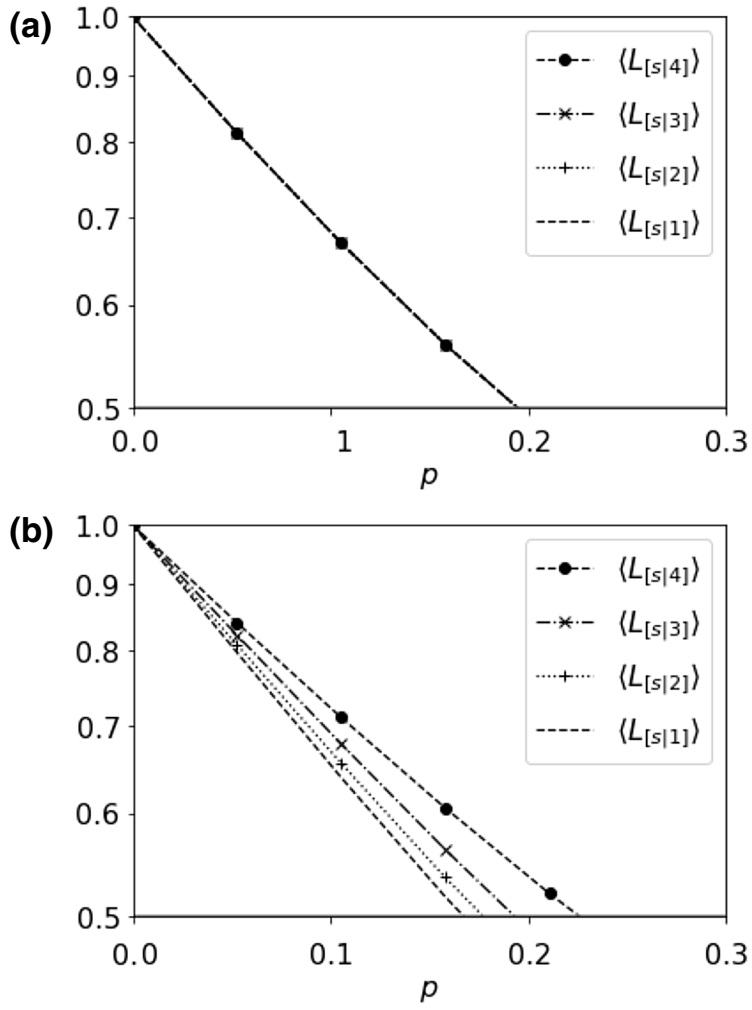

FIG. 11. Expectation values of the conditional test operator of Eq. (41) for four conditional bipartitions when the phenomenological depolarizing noise model (top panel) or the two-qubit depolarizing noise model (bottom panel) is applied to the non-FT readout of the plaquette. The $y$ axes are represented in log-scale whilst the $x$ axes represent the different error probabilities of the two depolarizing error models. In both cases the measurement error probability $p_{\mathrm{ME}}$ is assumed to be zero. In the bottom panel we assume an error-growth rate per gate of $r=0.2$. For the phenomenological depolarizing noise, due to the full symmetry of the error channel and the test operator with respect to different conditional bipartitions, the choice of the conditional bipartition is irrelevant. For the two-qubit depolarizing noise, on the other hand, the lowest performance belongs to the qubit pair that are involved in the first two-qubit gate, i.e., $[s \mid 1]$ (see the main text).

an ideal entangled logical state projector onto the ideal target state, Eq. (31), requiring an exponential number of measurements, (ii) efficient witnessing using the second SL witness method proposed in Ref. [29] requiring in this case a linear number of measurements, and (iii) conditional GME witnessing requiring a linear number of witnesses and measurements.

As discussed above, the six-qubit FT flag-based circuit presented in Fig. 9 is the trapped-ion version of the circuit presented in Fig. 3(d) compiled to the particular set of trapped-ion native gates (see Fig. 6). The role of the flag qubit in FTQEC circuits is to detect cascades of errors from the syndrome qubit to the data qubits after measuring it and extracting the syndrome. In the majority of cases the flag remains disentangled from the syndrome and therefore from the rest of data qubits. However, under some circumstances, such as coherent-rotation syndrome errors of the form $\exp (i \phi X)$, the entire plaquette will end up in an entangled state of Eq. (31). We remark here that, in this case, we artificially introduce the coherent rotation error to create six-qubit GME and thus, to qualitatively characterize the FT six-qubit QEC building block. Proving six-qubit genuine multipartite entanglement is thereby a way to guarantee that the expected quantum correlations between data, syndrome and flag qubits are built up, and that consequently, such dangerous errors propagate as expected through the circuit and can therefore be detected and corrected for.

The ideal six-qubit GME output state of Eq. (31) can now be used to evaluate the experimental quality of the plaquette measurement circuit. Beginning with the standard witnessing, to show that this state is GME, we check the entanglement in all 31 possible bipartitions. Let us focus on one of them, say $(f \mid s, 1,2,3,4)$, where we use the symbol $f$ for the flag qubit and $s$ for the the syndrome qubit. It is easy to see that, for instance, setting $\phi=\pi / 4$ the output state can be read off as the following bipartite state:

$$
\left|\psi_{\text {out }}\right\rangle=\frac{\left|\mathrm{GHZ}_{+}^{\otimes 5}\right\rangle|+\rangle_{f}+\left|\widetilde{\mathrm{GHZ}}_{+}^{\otimes 5}\right\rangle|-\rangle_{f}}{\sqrt{2}},
$$

where we denote $\left|\widetilde{\mathrm{GHZ}}_{+}^{\otimes 5}\right\rangle=(|00111\rangle-|11000\rangle) / \sqrt{2}$ with $\left\langle\mathrm{GHZ}_{+}^{\otimes 5} \mid \widetilde{\mathrm{GHZ}}_{+}^{\otimes 5}\right\rangle=0$. Consider now the test operator

$$
L=\left|\psi_{\text {out }}\right\rangle\left\langle\psi_{\text {out }}\right| \text {. }
$$

For the separability bound with respect to this bipartition, we are effectively dealing with a two-qubit system again, which allows us to obtain

$$
l_{f \mid s, 1,2,3,4}=\sup _{\sigma \in \mathcal{S} e p_{f} \mid s, 1,2,3,4} \operatorname{Tr}(L \sigma)=\frac{1}{2} .
$$

We note, however, that with respect to different bipartitions we may get different bounds. For each of the 31 bipartitions we evaluate the bounds and exhaustively list them in Table I of Appendix B. In each case, we either get a bound of $1 / 2$ or $1 / 4$. In the standard GME witnessing approach using a single test operator we must choose the largest value [see Eq. (21)], which, in this case, corresponds to $1 / 2$. Consequently, we can define a GME entanglement witness as

$$
W=\frac{1}{2} I-L,
$$

so that $\operatorname{Tr}(W \sigma) \geq 0$ for all states that are separable with respect to at least one of the bipartitions, while there exist 

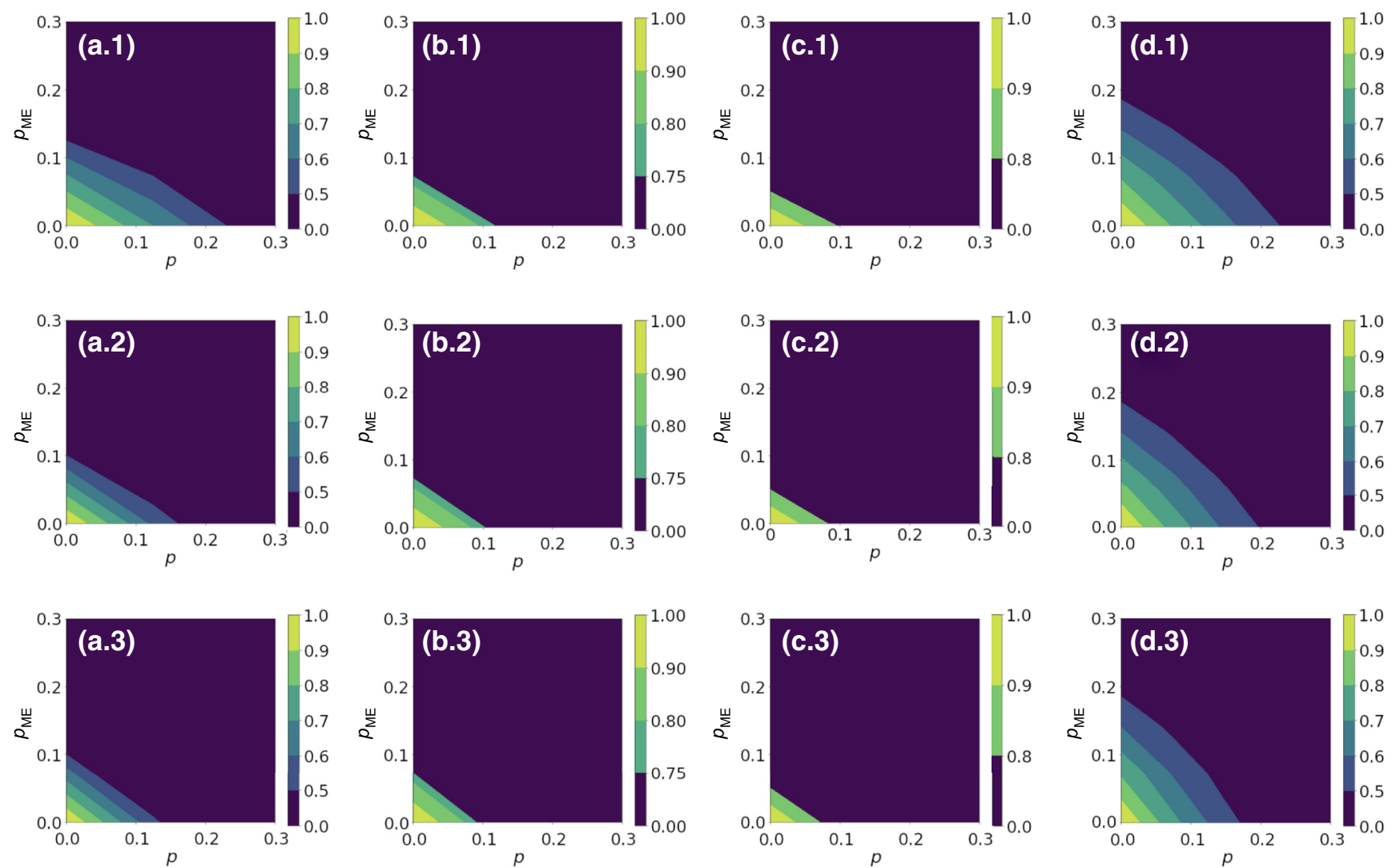

FIG. 12. GME witnessing plots in a five-qubit non-FT plaquette readout circuit subject to the two-qubit depolarizing noise model. In each row $i(i=1,2,3)$ the contour plots represent, for different values of depolarizing-noise probability $p$ and measurement-error probability $p_{\mathrm{ME}}$, the expectation values of (a.i) the standard GME witness $L_{\mathrm{GHZ} \otimes 5}$ in Eq. (38), (b.i) the first SL witness $L_{\mathrm{TG} 1}$ in Eq. (39), (c. $i)$ the second SL witness $L_{\mathrm{TG} 2}$ in Eq. (40), and (d.i) the worst-case conditional witnessing $L_{[s \mid x]}$ of Eq. (41) for $x=1$. In each row $i=1,2$, and 3 , the error-growth rate per gate values are $r=0,0.1$, and 0.2 , respectively. The colormap sidebars represent the witness bounds for each method. In all cases, the dark blue region identifies the noise values for which the GME tests are inconclusive.

quantum states for which $\operatorname{Tr}(W \varrho)<0$. Given any state $\varrho$ an expectation value $\operatorname{Tr}(\varrho L)>1 / 2$ thus implies the entanglement of $\varrho$ with respect to all bipartitions, i.e., its GME.

In Eq. (6), it is shown that a GHZ-like projector can be written in terms of its $2^{n}$ stabilizers with $n$ the number of physical qubits. Following the same procedure for the state in Eq. (44), the expectation value of the six-qubit test operator is

$$
\langle L\rangle=\operatorname{Tr} \varrho L=\frac{1}{2^{6}} \sum_{i=1}^{2^{6}}\left\langle S_{i}\right\rangle,
$$

where the stabilizer subgroup is now given by

$$
\begin{aligned}
\mathcal{S}_{6 q}= & \left\langle g_{1}=Z_{1} X_{3} X_{4} X_{5} Z_{6}, g_{2}=Z_{2} X_{3} X_{4} X_{5} Z_{6},\right. \\
& g_{3}=Z_{3} Z_{5}, g_{4}=Z_{4} Z_{5}, \\
& \left.g_{5}=-X_{1} X_{2} Z_{5} Z_{6}, g_{6}=X_{3} X_{4} Y_{5} Y_{6}\right\rangle .
\end{aligned}
$$

For the standard witnessing of GME using the six-qubit projector $L$, it is thus sufficient to locally measure the stabilizers at the output of the plaquette circuit, combine their statistics according to Eq. (48), and verify that $\langle L\rangle>1 / 2$.

The second approach we consider is the SL witnessing of GME [29]. In this occasion, the first SL test operator is not applicable to the six-qubit state in Eq. (44) due to the lack of required symmetries in stabilizer generators in Eq. (49). The simplest witness we can get is by summing up all the generators as in the second SL witness

$$
L_{\mathrm{TG} 2}=\frac{1}{6} \sum_{i=1}^{6} g_{i} .
$$

The GME bound for this witness is $l_{\mathrm{TG} 2}=5 / 6$ [29]. In contrast to the five-qubit case, due to the form of the sixqubit stabilizer generators, this witness would require more than two, yet a linear number of, measurement settings.

Finally, we implement our conditional GME witness. Using Theorem 1, we need to test five conditional bipartitions $[f \mid 1],[f \mid 2],[f \mid 3],[f \mid 4]$, and $[f \mid s]$. In this case, we need to distinguish two different conditional test operators 
to cover all the five conditional bipartitions. We introduce the first set of conditional test operators as,

$$
L_{[f \mid x]}^{1}=|\widetilde{\mathrm{Bell}}\rangle_{f \mid x}\langle\widetilde{\mathrm{Bel} l}|\otimes| 0\rangle_{1,2}\left\langle\left. 0\right|^{\otimes 2} \otimes \mid+\right\rangle_{\{f, x, 1,2\}^{\mathrm{c}}}\left\langle+\left.\right|^{\otimes 2}\right.
$$

for $x \in\{3,4, s\}$, in which $|\widetilde{\mathrm{Bell}}\rangle_{f \mid x}\langle\widetilde{\mathrm{Bell}}|$ is the projection of the qubit pair $f$ and $x$ onto the Bell state $|\widetilde{\mathrm{Bell}}\rangle=$ $(|0+\rangle+|1-\rangle) / \sqrt{2}$. This conditioning choice is not applicable to $x \in\{1,2\}$ since it would not lead to any Bell state including the flag qubit. Thus, we use a second set of conditional test operators for the remaining $x \in\{1,2\}$ conditional bipartitions,

$$
L_{[f \mid x]}^{2}=|\widetilde{\mathrm{Bel} l}\rangle_{f \mid x}\langle\widetilde{\mathrm{Bel} l}|\otimes|+\rangle_{\{f, s, x, 3,4\}^{\mathrm{c}}}\langle+|\otimes| 0\rangle_{s, 3,4}\left\langle\left. 0\right|^{\otimes 3}\right.
$$

As the conditioned two-qubit states are identical for both test operators $L_{[f \mid x]}^{1}$ and $L_{[f \mid x]}^{2}$, the bounds are the same,

$$
l_{f \mid x}^{1,2}=\sup _{\sigma \in \mathcal{S} e p_{f} \mid x} \operatorname{Tr}\left(\sigma|\widetilde{\mathrm{Bell}}\rangle_{f \mid x}\langle\widetilde{\mathrm{Bel} l}|\right)=\frac{1}{2},
$$

where the supremum is taken over the set of all bipartite separable states of qubits $f$ and $x$. Consequently, for any conditional bipartition $[f \mid x]$ with $x \in\{1,2,3,4, s\}$ and any given six-qubit state $\varrho$, an expectation value satisfying $\left\langle L_{\left[f^{\prime} \mid x\right]}^{1,2}\right\rangle>1 / 2$ for all $x \in\{1,2,3,4, s\}$ implies GME of the quantum state $\varrho$. Importantly for us, the $|\widetilde{B e l l}\rangle$ state is the one-dimensional code space of the stabilizer generators $\{X Z, Z X\}$,

$$
|\widetilde{\mathrm{Bel}}\rangle_{f \mid x}\langle\widetilde{\mathrm{Bel}}|=\frac{I_{f} I_{x}+X_{f} Z_{x}+Z_{f} X_{x}+Y_{f} Y_{x}}{4},
$$

implying that conditional GME witnessing can be performed solely by local Pauli measurements on qubits.
Furthermore, for the implementation of conditional GME witnessing we need three settings per bipartition, hence a total of 15 measurement settings.

\section{Phenomenological depolarizing noise model}

The contour plots of Fig. 13 present the results of the three witnessing techniques with the four test operators to the six-qubit FT plaquette that suffers from the circuit noise of Eq. (32) and the measurement noise of Eq. (36) for a coherent error angle of $\phi=\pi / 4$. From left to right, the four columns of subfigures represent the results obtained for (a) the standard witness of Eq. (48), (b) the efficient witness of Eq. (50), (c) our conditional witness of Eq. (51) for $x=s$ and (d) the conditional witness of Eq. (52) for $x=2$. For this error model the expectation values of $L_{[f \mid x]}^{1}$ and $L_{[f \mid x]}^{2}$ behave equally for $x=3,4, s$ and $x=1,2$, respectively; see the top panel of Fig. 15. This is due to the symmetry of the error channel with respect to each individual qubit, hence our choices of $x$ in Figs. 13(c) and 13(d). In all plots the dark blue shaded areas represent states for which tests result values below the separability bounds and no GME is detected. As we can see, the increase in the noise tolerance of conditional GME witnessing compared to the standard methods is remarkable.

Setting $\phi=\pi / 4$, we find that for $p_{\mathrm{ME}}=0$ our conditional witnessing method shows the highest robustness against the depolarizing noise up to $p \approx 0.21$ and $p \approx 0.26$ as shown in Figs. 13(c) and 13(d), respectively. With the standard method [Fig. 13(a)] the noise resilience drops to $p \approx 0.11$ and its lowest value is seen for the second SL method [Fig. 13(b)] at $p \approx 0.04$. Here again, we clearly observe that linearizing the number of measurements typically results in a reduction of the robustness. Similarly, by fixing the probability of depolarizing error to $p=0$, one notices that the measurement-error probability $p_{\mathrm{ME}}$ also reaches its highest threshold value for our conditional
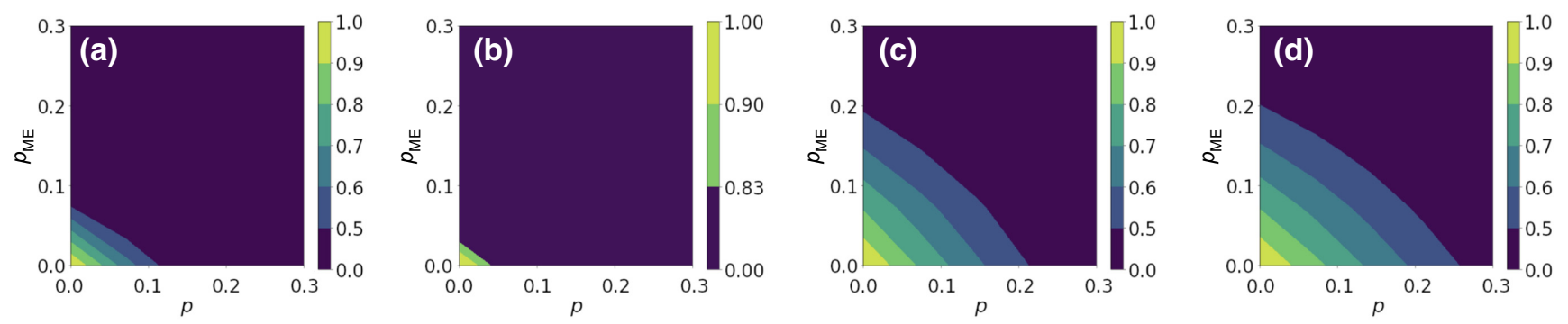

FIG. 13. GME witnessing plots in a six-qubit flag-based FT plaquette readout circuit under the phenomenological depolarizing noise model of Sec. VIB 1. The coherent-noise angle is set to $\phi=\pi / 4$. Each contour plot represents, for different values of depolarizingnoise probability $p$ and measurement-error probability $p_{\mathrm{ME}}$, the expectation values of (a) the standard GME witness $L$ of Eq. (48), (b) the second SL witness, $L_{\mathrm{TG} 2}$ in Eq. (50), (c) the worst-case conditional witnessing using $L_{[f \mid x]}^{1}$ for $x=s$ from Eq. (51), and (d) the worst-case conditional witnessing using $L_{[f \mid x]}^{2}$ in Eq. (52) for $x=2$. The colormap sidebars represent the witness bounds for each method. In all cases, the dark blue region identifies the noise values for which the GME tests are inconclusive. 

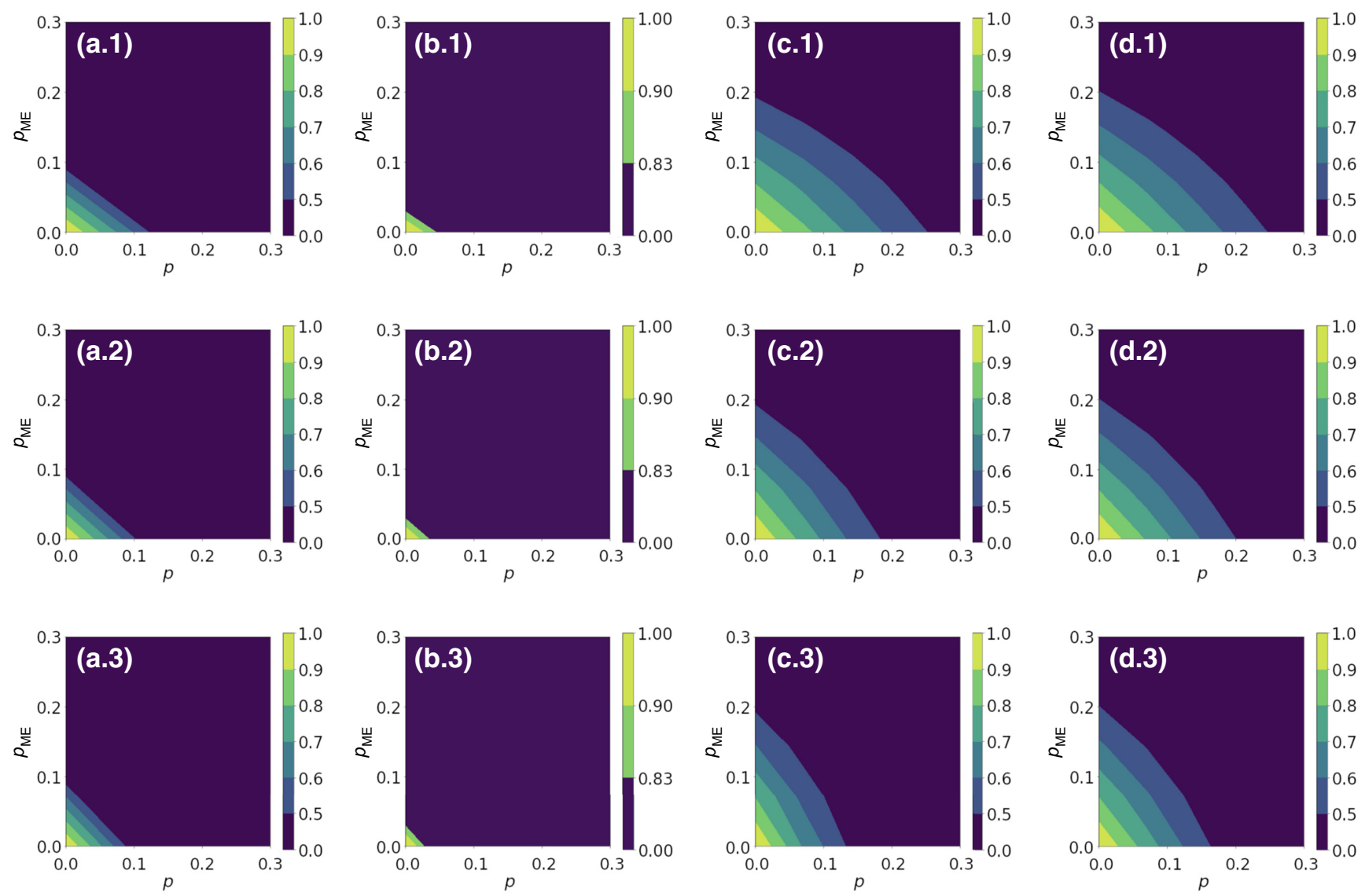

FIG. 14. GME witnessing plots in a six-qubit flag-based FT plaquette subject to the two-qubit depolarizing noise model of Sec. VIB 2. The coherent-noise angle is set to $\phi=\pi / 4$. In each row $i(i=1,2,3)$ the contour plots represent, for different values of depolarizing-noise probability $p$ and measurement-error probability $p_{\mathrm{ME}}$, the expectation values of (a.i) $L$ in the standard GME witness $L$ in Eq. (48), (b.i) the second SL witness $L_{\mathrm{TG} 2}$ in Eq. (50), (c. $i$ ) the worst-case conditional witnessing using $L_{[f \mid x]}^{1}$ for $x=s$ from Eq. (51) and (d.i) the worst-case conditional witnessing using $L_{[f \mid x]}^{2}$ in Eq. (52) for $x=2$. In each row $i=1,2$, and 3, the error-growth rate per gate values are $r=0,0.1$, and 0.2 , respectively. The colormap sidebars represent the witness bounds for each method. In all cases, the dark blue region identifies the noise values for which the GME tests are inconclusive.

witnessing methods both close to 0.2 . For the standard witnessing method we get a threshold of $p_{\mathrm{ME}} \approx 0.08$ whilst it reaches its lowest values for the second SL witness at $p_{\mathrm{ME}} \approx 0.04$.

\section{Two-qubit depolarizing noise model}

We now assume that the six-qubit FT plaquette suffers from the more realistic circuit noise of Eq. (33), which applies a two-qubit depolarizing channel after each entangling gate. As before, we also include the measurement noise effects as per Eq. (36). Figure 14 represents the resulting contour plots of the expectation values for $\phi=$ $\pi / 4$ of (a) the standard witness of Eq. (48), (b) the SL witness of Eq. (50), (c) our conditional witness of Eq. (51) for $x=s$, and (d) our conditional witness of Eq. (52) for $x=2$. The conditional bipartitions $[f \mid s]$ and $[f \mid 2]$ chosen here are the ones in which the conditional GME witnessing performs the worst as shown in the bottom panel of Fig. 15.
We observe that with increasing error-growth rate $r$ the threshold for $p$ decreases. However, even for the worstcase scenario, the conditional GME witnessing represented in Figs. 14(c) and 14(d) show significant robustness compared to the standard witnesses for each $r$. By comparing the effects of the two different noise models shown in Figs. 13 and 14, one notices again that the more realistic two-qubit depolarizing noise results in higher thresholds of detectable GME states than the phenomenological noise. Within both error models, the conditional GME witnessing methods are not just more efficient in the number of measurement settings, but also more robust against errors compared to their standard counterparts.

\section{CONCLUSIONS AND OUTLOOK}

We introduce conditional entanglement witnessing as a robust and efficient technique to test GME in multipartite quantum systems. We prove that, to show GME 
(a)

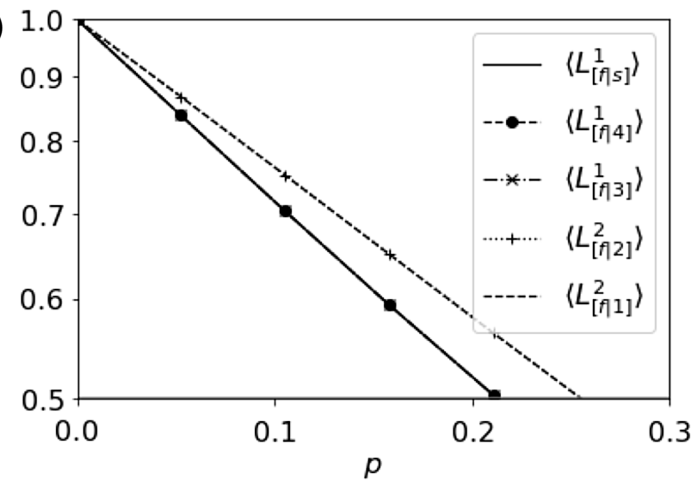

(b)

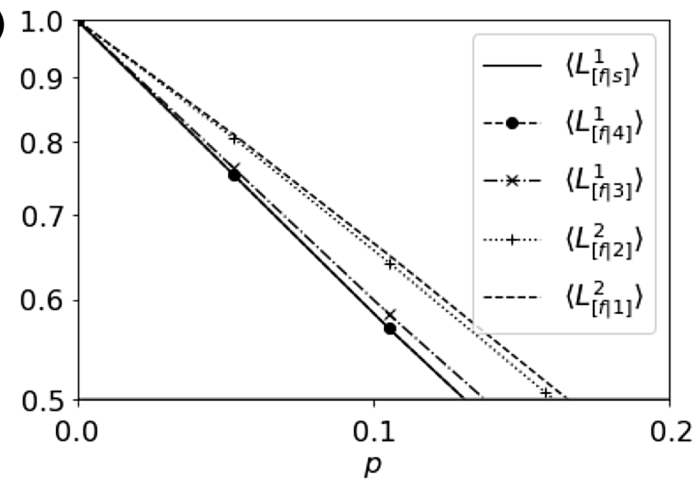

FIG. 15. Expectation values of the conditional test operators of Eqs. (51) and (52) for five conditional bipartitions for the phenomenological depolarizing noise model (top panel) and for the two-qubit depolarizing noise model (bottom panel) applied to the flag-based FT plaquette. The $y$ axes are represented in log scale whilst the $x$ axes represent the different error probabilities of the two depolarizing error models. In both cases the measurementerror probability $p_{\mathrm{ME}}$ is assumed to be zero. In the bottom panel we assume an error-growth rate per gate of $r=0.2$. For both noise models, the lowest performance of the test operators $L_{[f \mid x]}^{1}$ and $L_{[f \mid x]}^{2}$ corresponds to $x=s$ and $x=2$, respectively.

in a $n$-partite system it is sufficient to conditionally verify entanglement in just $n-1$ conditional bipartitions. We then introduce the conditional GME witnessing technique and apply it to non-FT and FT readout circuits for stabilizer operators. This enables us to characterize the performance of these circuits as building blocks of larger topological QEC codes in terms of their power for generating GME entanglement.

In particular, we pick a $g_{x}$ syndrome-readout plaquette of the $d=3$ topological color code and its flag-based FT version adapted to a shuttling-based trapped-ion platform. We demonstrate the efficiency and robustness of the conditional witnessing compared to the standard witnessing method when the plaquette circuit undergoes phenomenological and two-qubit gate depolarizing noise and measurements suffer from bit-flip errors. In terms of efficiency, for the five-qubit non-FT scheme, in general, the number of measurement settings needed in the standard fidelity witnessing is 31, whilst the SL approach of Ref. [29] needs two settings, at the expense of a higher fragility in the presence of noise. Our conditional witnessing requires 12 settings, but we note that its growth remains linear in the number of qubits in contrast to the exponentially increasing complexity of the fidelity witness.

For the six-qubit FT scheme, the number of measurement settings for the standard fidelity witness rises to 63 whereas our conditional witnessing requires only 15 where one can already observe the exponential versus linear advantage. In this case, one SL witness worked at the cost of a significant drop in the noise robustness. With regards to the robustness of these methods, the conditional GME witness hereby proposed yields a significant increase in the noise tolerance compared to both the standard fidelity estimate, and SL witnesses.

We are convinced that, with the current technology where the addition of each qubit amplifies the unavoidable noise and errors, it is beneficial to use our conditional entanglement witnessing scheme to certify the entangling power of quantum processors wherein the output states are generic mixed states far from ideal expected output states. We note that certification of entanglement can also help benchmark building blocks and gadgets of logical qubits and gate operations, as demonstrated for trapped ions in recent works [94,95], towards faulttolerant quantum computation. Moreover, our witnessing technique can be applied to any multipartite quantum system, independently of the nature of its qubits, as long as sufficient control (single- and two-qubit gates, Pauli measurements) is available. It can therefore be applied to benchmark the capabilities of entanglement generation in other quantum-processing platforms, including superconducting qubits [96], neutral atoms [97], spin qubits [98], Nitrogen-Vacancy centers [99], and photonic systems [100]. Lastly, it can be used in a broad variety of protocols where efficient entanglement certification plays an important role [101-104]. Last but not least, an interesting direction for further research is to evaluate and compare the performance of the witnessing methods discussed here under more refined microscopic noise models.

\section{ACKNOWLEDGMENTS}

We acknowledge fruitful discussions with experimental colleagues J. Hilder, D. Pijn, U. Poschinger, and F. Schmidt-Kaler from Johannes Gutenberg Universität Mainz, Germany, as well as with colleagues from the eQual and AQTION collaborations. We gratefully acknowledge support by the EU Quantum Technology Flagship Grant AQTION 820495. A.R.B. acknowledges support by the Universidad Complutense de MadridBanco Santander Predoctoral Fellowship. A.B. acknowledges support from the Ramón y Cajal Program RYC2016-20066, and CAM/FEDER Project S2018/TCS- 4342 (QUITEMAD-CM) and the Plan Nacional Generación de 
Conocimiento PGC2018-095862-B-C22. M.M. acknowledges support by the ERC Starting Grant QNets 804247, and also by U.S.A.R.O. through Grant No. W911NF-14-1010. F.S. acknowledges support and resources provided by the Royal Commission for the Exhibition of 1851.

\section{APPENDIX A: $Z$-TYPE PARITY-CHECK CIRCUIT WITH CNOT GATES}

Here we provide the $Z$-type parity-check circuits in parallel with the $X$-type parity check of Sec. III. It is assumed that the circuits are ideal and free from state preparation and measurement and gate errors. We provide the output states from these circuits and their stabilizer generators from which all the entanglement witnesses presented in the text can be obtained.

\section{Non-FT circuit}

Starting by the ideal plaquette circuit depicted in Fig. 16(a) representing the non-FT $g_{z}$ readout circuit, we initialize the data qubits $\left\{i_{1}, i_{2}, i_{3}, i_{4}\right\}$ in $|+\rangle^{\otimes 4}$ and the syndrome qubit in $|0\rangle_{s}$. This is expected to produce the output state

$$
\left|\psi_{\text {out }}\right\rangle=\left|\mathrm{GHZ}_{X+}^{\otimes 5}\right\rangle=\frac{|+\rangle^{\otimes 5}+|-\rangle^{\otimes 5}}{\sqrt{2}} .
$$

The resulting five-qubit GHZ state (in the $X$ basis) in Eq. (A1) can be understood as the one-dimensional code space of the stabilizer subgroup

$$
\begin{aligned}
\mathcal{S}_{X, 5 q} & =\left\langle g_{1}=X_{1} X_{2}, g_{2}=X_{2} X_{3}, g_{3}=X_{3} X_{4},\right. \\
g_{4} & \left.=X_{4} X_{5}, g_{5}=Z_{1} Z_{2} Z_{3} Z_{4} Z_{5}\right\rangle .
\end{aligned}
$$

\section{Flag-based FT circuit}

We now consider the FT version of the $Z$-type parity check by adding a flag qubit as shown in Fig. 16(b). As in the previous case, the data and the syndrome qubits are initialized in $|+\rangle^{\otimes 4}$ and $|0\rangle_{s}$, respectively, whereas the flag qubit is prepared in $|+\rangle_{f}$. The expected output state is then given by

$$
\left|\psi_{\text {out }}\right\rangle=\frac{\left(|+\rangle^{\otimes 5}+|-\rangle^{\otimes 5}\right)|+\rangle_{f}}{\sqrt{2}}=\left|\mathrm{GHZ}_{X+}^{\otimes 5}\right\rangle|+\rangle_{f} .
$$

As discussed in the main text, in this scheme the flag qubit will detect the presence of a single error on the syndrome qubit that propagates to multiple errors on the data qubits, thus enabling the preservation of the encoded information. For example, if a single Pauli $Z$ error occurs between the second and the third data-to-syndrome CNOT gates in
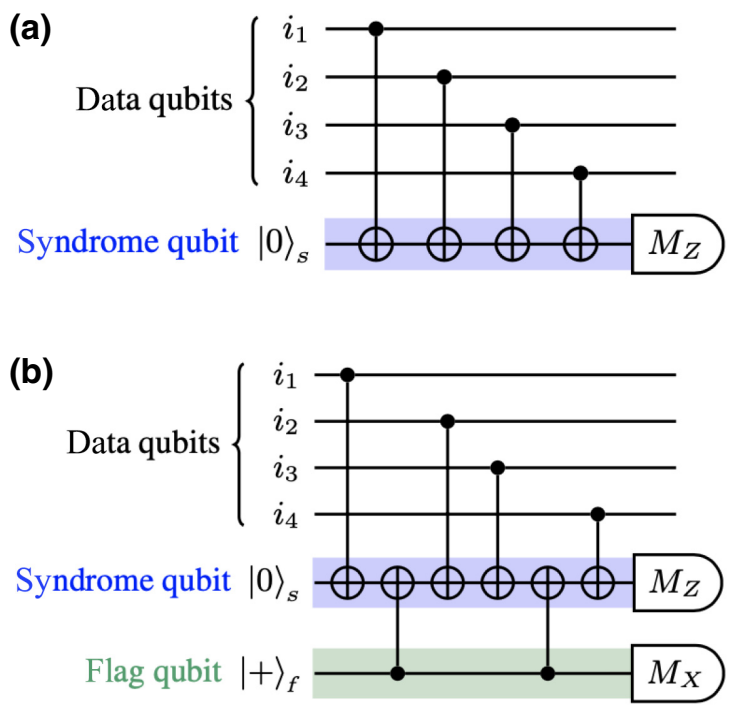

FIG. 16. Error syndrome extraction circuits in the color code for (a) the non-FT and (b) the flag-based FT readouts of the generators $g_{z}^{(q)}$ [Eq. (3)] of the plaquette $q$ in Fig. 1. The data qubits $i_{1}, i_{2}, i_{3}, i_{4}$ correspond to the four physical qubits of this plaquette.

Fig. 16(b), it leads to the state

$$
\left|\psi_{\text {out }}\right\rangle=Z_{s} Z_{3} Z_{4}\left|\mathrm{GHZ}_{X+}^{\otimes 5}\right\rangle|-\rangle_{f},
$$

where the flag has been flipped. A final projective measurement on the flag qubit thus reveals the error.

\section{APPENDIX B: EXHAUSTIVE LIST OF SEPARABILITY BOUNDS FOR STANDARD WITNESSING IN THE FLAG-BASED FT CIRCUIT}

In Sec. VI B we use the test operator

$$
L=\left|\psi_{\text {out }}\right\rangle\left\langle\psi_{\text {out }}\right|
$$

wherein

$$
\left|\psi_{\text {out }}\right\rangle=\frac{\left|\mathrm{GHZ}_{+}^{\otimes 5}\right\rangle|+\rangle_{f}+\left|\widetilde{\mathrm{GHZ}}_{+}^{\otimes 5}\right\rangle|-\rangle_{f}}{\sqrt{2}},
$$

with $\left|\widetilde{\mathrm{GHZ}}_{+}^{\otimes 5}\right\rangle=(|00111\rangle-|11000\rangle) / \sqrt{2}$. Here, we give the exhaustive list of separability bounds for $L$,

$$
l_{B}=\sup _{\sigma \in \mathcal{S e p}_{B}} \operatorname{Tr}(L \sigma),
$$

with respect to each bipartition $B$ within the set of all 31 possible bipartitions $\mathcal{B}$ and show that they are either $1 / 2$ or $1 / 4$.

In the most general form, the solution to Eq. (B3) is obtained by solving a set of coupled nonlinear eigenvalue equations called multipartite separability eigenvalue 
TABLE I. Separability values for the test operator of Eq. (45) with respect to all 31 bipartitions.

\begin{tabular}{lc}
\hline \hline Bipartition $B$ & \\
\hline$(f \mid s, 1,2,3,4)$ & $1 / 2$ \\
$(s \mid f, 1,2,3,4)$ & $1 / 2$ \\
$(1 \mid f, s, 2,3,4)$ & $1 / 2$ \\
$(2 \mid f, s, 1,3,4)$ & $1 / 2$ \\
$(3 \mid f, s, 1,2,4)$ & $1 / 2$ \\
$(4 \mid f, s, 1,2,3)$ & $1 / 2$ \\
$(f, s \mid 1,2,3,4)$ & $1 / 4$ \\
$(f, 1 \mid s, 2,3,4)$ & $1 / 4$ \\
$(f, 2 \mid s, 1,3,4)$ & $1 / 4$ \\
$(f, 3 \mid s, 1,2,4)$ & $1 / 4$ \\
$(f, 4 \mid s, 1,2,3)$ & $1 / 4$ \\
$(s, 1 \mid f, 2,3,4)$ & $1 / 4$ \\
$(s, 2 \mid f, 1,3,4)$ & $1 / 4$ \\
$(s, 3 \mid f, 1,2,4)$ & $1 / 2$ \\
$(s, 4 \mid f, 1,2,3)$ & $1 / 2$ \\
$(1,2 \mid f, s, 3,4)$ & $1 / 2$ \\
$(1,3 \mid f, s, 2,4)$ & $1 / 4$ \\
$(1,4 \mid f, s, 2,3)$ & $1 / 4$ \\
$(2,3 \mid f, s, 1,4)$ & $1 / 4$ \\
$(2,4 \mid f, s, 1,3)$ & $1 / 4$ \\
$(3,4 \mid f, s, 1,2)$ & $1 / 2$ \\
$(f, s, 1 \mid 2,3,4)$ & $1 / 4$ \\
$(f, s, 2 \mid 1,3,4)$ & $1 / 4$ \\
$(f, s, 3 \mid 1,2,4)$ & $1 / 4$ \\
$(f, s, 4 \mid 1,2,3)$ & $1 / 4$ \\
$(f, 1,2 \mid s, 3,4)$ & $1 / 2$ \\
$(f, 1,3 \mid s, 2,4)$ & $1 / 4$ \\
$(f, 1,4 \mid s, 2,3)$ & $1 / 4$ \\
$(f, 2,3 \mid s, 1,4)$ & $1 / 4$ \\
$(f, 2,4 \mid s, 1,3)$ & $1 / 4$ \\
$(f, 3,4 \mid s, 1,2)$ & \\
\hline \hline &
\end{tabular}

equations $[26,48]$. This is a highly nontrivial problem to solve in general. Nevertheless, in some cases, including the example at hand, we can obtain the bounds without directly solving these equations and by merely relying on the properties of $\left|\psi_{\text {out }}\right\rangle$. In particular, the property we use here is the following. Consider a bipartite pure state of the form

$$
|\psi\rangle_{A B}=\frac{1}{\sqrt{n}} \sum_{i=1}^{n}|i\rangle_{A}\left|\psi_{i}\right\rangle_{B},
$$

such that $\langle i \mid j\rangle=\delta_{i j}$ and $\left\langle\psi_{i} \mid \psi_{j}\right\rangle=\delta_{i j}$. Using techniques from Refs. [26,48], we infer that the maximum separability bound is

$$
l_{A B}=\sup _{\sigma \in \mathcal{S} e p_{A B}} \operatorname{Tr}|\psi\rangle\langle\psi| \sigma=\frac{1}{n} .
$$

In other words, biorthogonality properties of the local Schmidt bases allow us to read the separability values off the Schmidt rank of $|\psi\rangle$. It turns out that we can use this result in the specific case of $\left|\psi_{\text {out }}\right\rangle$ in Eq. (B2). We skip

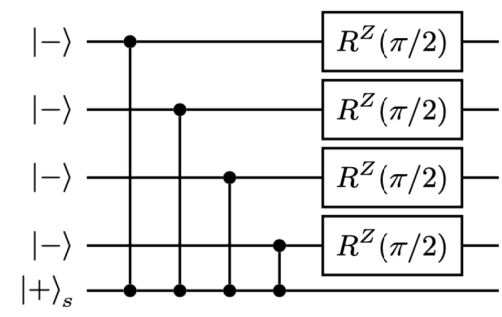

FIG. 17. The non-FT circuit using the native trapped-ion gates.

writing down Schmidt decompositions with respect to individual bipartitions, as this is straightforward, and merely list the separability bounds in Table I. Nevertheless, to give an example, we notice that $\left|\psi_{\text {out }}\right\rangle$ in Eq. (B2) is written in its Schmidt decomposition with respect to the bipartition $(f \mid s, 1,2,3,4)$ with an Schmidt rank of 2 and it is of the form of Eq. (B4), hence $l_{f \mid s, 1,2,3,4}=1 / 2$.

\section{APPENDIX C: $Z$-TYPE PARITY-CHECK CIRCUITS USING NATIVE TRAPPED-ION GATES}

We now translate the $Z$-type parity-check circuits of Appendix A into the trapped ions' native gates using the relations given in Fig. 6. We also provide the output states from these circuits and their stabilizer generators by means of which the entanglement witnesses given in the main text can be constructed.

\section{Non-FT circuit}

In Fig. 17 the circuit for $Z$-type syndrome readout using the native trapped-ion language is shown. We start by initializing the data qubits in $|-\rangle^{\otimes 4}$ and the syndrome qubit in $|+\rangle_{s}$. Similar to the CNOT-based circuit, this is expected to produced the output state

$$
\left|\psi_{\text {out }}\right\rangle=\left|\mathrm{GHZ}_{X+}^{\otimes 5}\right\rangle=\frac{|+\rangle^{\otimes 5}+|-\rangle^{\otimes 5}}{\sqrt{2}},
$$

with the same stabilizer generators as given in Eq. (A2). This means that $\left|\psi_{\text {out }}\right\rangle$ in Eq. (C1) is a +1 eigenstate of the five generating stabilizers $g_{i}$ from Eq. (A2) and all their possible combinations.

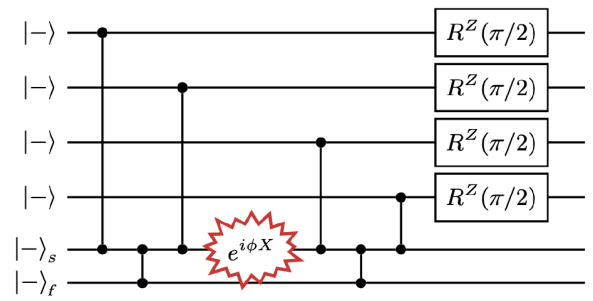

FIG. 18. The flag-based FT circuit using the native trapped-ion gates. The $e^{i \phi X}$ error is injected to create a six-qubit entangled state in the flag-based FT stabilizer measurement. 


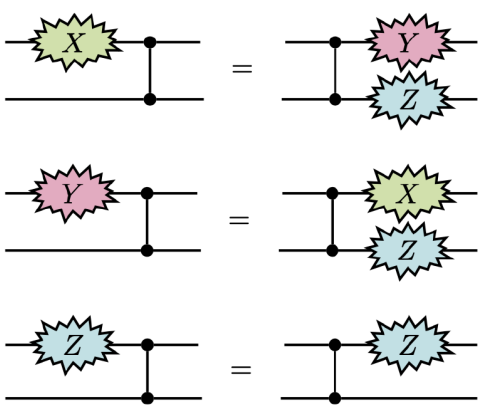

FIG. 19. Error propagation through $Z Z$ gates. A Pauli $X(Y)$ error propagates as a $Y(X)$ error and a $Z$ error onto the second qubit. An incoming single $Z$ error commutes with the $Z Z$ gate and therefore does not propagate onto the second qubit.

\section{Flag-based FT circuit}

Here, we present the flag-based $g_{z}$ readout circuit using the trapped-ion native gate set. Recall that, to generate entanglement between the flag, syndrome, and data qubits we need to inject an error of the form $\exp (i \phi X)$ in a controllable way; see Fig. 18. In this occasion we initialize the data qubits in $|-\rangle^{\otimes 4}$, the syndrome in $|-\rangle_{s}$, and the flag qubit in $|-\rangle_{f}$. The output state is then

$$
\begin{aligned}
\left|\psi_{\text {out }}\right\rangle= & \cos \phi|+\rangle_{f}\left|\mathrm{GHZ}_{X+}^{\otimes 5}\right\rangle \\
& +i \sin \phi|-\rangle_{f} Y_{s} Z_{3} Z_{4}\left|\mathrm{GHZ}_{X+}^{\otimes 5}\right\rangle,
\end{aligned}
$$

where the stabilizer subgroup is given by

$$
\begin{aligned}
\mathcal{S}_{\tilde{X}, 6 q}= & \left\langle g_{1}=X_{1} Z_{3} Z_{4} Z_{5} Z_{6}, g_{2}=X_{2} Z_{3} Z_{4} Z_{5} Z_{6},\right. \\
& g_{3}=X_{3} X_{5}, g_{4}=X_{4} X_{5}, \\
& \left.g_{5}=-Z_{1} Z_{2} X_{5} Z_{6}, g_{6}=-Z_{3} Z_{4} Y_{5} Y_{6}\right\rangle .
\end{aligned}
$$

\section{APPENDIX D: ERROR PROPAGATION THROUGH $Z Z$ GATES}

In Fig. 19 we give the Pauli-error propagation rules through the $Z Z$-entangling gates of trapped-ion platforms. These come in handy in calculating the output states, e.g., that of Eq. (31).

[1] T. D. Ladd, F. Jelezko, R. Laflamme, Y. Nakamura, C. Monroe, and J. L. O'Brien, Quantum computers, Nature 464, 45 (2010).

[2] F. Arute et al., Quantum supremacy using a programmable superconducting processor, Nature 574, 505 (2019).

[3] D. Gottesman, Quantum fault tolerance in small experiments, arXiv:1610.03507 [quant-ph] (2016).

[4] A. Bermudez, X. Xu, R. Nigmatullin, J. O'Gorman, V. Negnevitsky, P. Schindler, T. Monz, U. G. Poschinger, C. Hempel, J. Home, F. Schmidt-Kaler, M. Biercuk, R. Blatt, S. Benjamin, and M. Müller, Assessing the Progress of Trapped-Ion Processors Towards Fault-Tolerant Quantum Computation, Phys. Rev. X 7, 041061 (2017).

[5] P. W. Shor, Scheme for reducing decoherence in quantum computer memory, Phys. Rev. A 52, R2493 (1995).

[6] A. R. Calderbank and P. W. Shor, Good quantum errorcorrecting codes exist, Phys. Rev. A 54, 1098 (1996).

[7] A. M. Steane, Error Correcting Codes in Quantum Theory, Phys. Rev. Lett. 77, 793 (1996).

[8] M. A. Nielsen and I. L. Chuang, Quantum Computation and Quantum Information (Cambridge University Press, Cambridge, 2000).

[9] F. Gaitan, Quantum Error Correction and Fault Tolerant Quantum Computing (CRC Press, Inc., USA, 2007).

[10] F. Schmidt-Kaler, S. Gulde, M. Riebe, T. Deuschle, A. Kreuter, G. Lancaster, C. Becher, J. Eschner, H. Häffner, and R. Blatt, The coherence of qubits based on single $\mathrm{Ca}^{+}$ions, J. Phys. B: At. Mol. Opt. Phys. 36, 623 (2003).

[11] M. Riebe, K. Kim, P. Schindler, T. Monz, P. O. Schmidt, T. K. Körber, W. Hänsel, H. Häffner, C. F. Roos, and R. Blatt, Process Tomography of ion Trap Quantum Gates, Phys. Rev. Lett. 97, 220407 (2006).

[12] J. M. Chow, J. M. Gambetta, L. Tornberg, J. Koch, L. S. Bishop, A. A. Houck, B. R. Johnson, L. Frunzio, S. M. Girvin, and R. J. Schoelkopf, Randomized Benchmarking and Process Tomography for Gate Errors in a Solid-State Qubit, Phys. Rev. Lett. 102, 090502 (2009).

[13] S. J. Beale and J. J. Wallman, Efficiently computing logical noise in quantum error correcting codes, arXiv:2003.10511 (2020).

[14] N. M. Linke, M. Gutierrez, K. A. Landsman, C. Figgatt, S. Debnath, K. R. Brown, and C. Monroe, Fault-tolerant quantum error detection, Sci. Adv. 3, e1701074 (2017).

[15] C. J. Trout, M. Li, M. Gutiérrez, Y. Wu, S.-T. Wang, L. Duan, and K. R. Brown, Simulating the performance of a distance-3 surface code in a linear ion trap, New J. Phys. 20, 043038 (2018).

[16] D. Nigg, M. Müller, E. A. Martinez, P. Schindler, M. Hennrich, T. Monz, M. A. Martin-Delgado, and R. Blatt, Quantum computations on a topologically encoded qubit, Science 345, 302 (2014).

[17] L. DiCarlo, M. D. Reed, L. Sun, B. R. Johnson, J. M. Chow, J. M. Gambetta, L. Frunzio, S. M. Girvin, M. H. Devoret, and R. J. Schoelkopf, Preparation and measurement of three-qubit entanglement in a superconducting circuit, Nature 467, 574 (2010).

[18] A. D. Córcoles, E. Magesan, S. J. Srinivasan, A. W. Cross, M. Steffen, J. M. Gambetta, and J. M. Chow, Demonstration of a quantum error detection code using a square lattice of four superconducting qubits, Nat. Commun. 6, 6979 (2015).

[19] C. K. Andersen, A. Remm, S. Lazar, S. Krinner, N. Lacroix, G. J. Norris, M. Gabureac, C. Eichler, and A. Wallraff, Repeated quantum error detection in a surface code, Nat. Phys. 16, 875 (2020).

[20] R. Jozsa and N. Linden, On the role of entanglement in quantum-computational speed-up, Proc. R. Soc. London Ser. A: Math. Phys. Eng. Sci. 459, 2011 (2003).

[21] L. Gurvits, in Proceedings of the Thirty-Fifth Annual ACM Symposium on Theory of Computing, STOC '03 
(Association for Computing Machinery, New York, NY, USA, 2003), p. 10.

[22] S. Gharibian, Strong NP-hardness of the quantum separability problem, Quantum Inf. Comput. 10, 343 (2010).

[23] M. Horodecki, P. Horodecki, and R. Horodecki, Separability of mixed states: Necessary and sufficient conditions, Phys. Lett. A 223, 1 (1996).

[24] B. M. Terhal, Bell inequalities and the separability criterion, Phys. Lett. A 271, 319 (2000).

[25] M. Lewenstein, B. Kraus, J. I. Cirac, and P. Horodecki, Optimization of entanglement witnesses, Phys. Rev. A 62, 052310 (2000).

[26] J. Sperling and W. Vogel, Multipartite Entanglement Witnesses, Phys. Rev. Lett. 111, 110503 (2013).

[27] R. Horodecki, P. Horodecki, M. Horodecki, and K. Horodecki, Quantum entanglement, Rev. Mod. Phys. 81, 865 (2009).

[28] Y. Zhou, Q. Zhao, X. Yuan, and X. Ma, Detecting multipartite entanglement structure with minimal resources, Npj Quantum Inf. 5, 83 (2019).

[29] G. Tóth and O. Gühne, Entanglement detection in the stabilizer formalism, Phys. Rev. A 72, 022340 (2005).

[30] M. Waegell and J. Dressel, Benchmarks of nonclassicality for qubit arrays, Npj Quantum Inf. 5, 66 (2019).

[31] O. Gühne and N. Lütkenhaus, Nonlinear Entanglement Witnesses, Phys. Rev. Lett. 96, 170502 (2006).

[32] F. Shahandeh, M. Ringbauer, J. C. Loredo, and T. C. Ralph, Ultrafine Entanglement Witnessing, Phys. Rev. Lett. 118, 110502 (2017).

[33] Y. Zhou, Entanglement detection under coherent noise: Greenberger-Horne-Zeilinger-like states, Phys. Rev. A 101, 012301 (2020).

[34] F. Verstraete, M. Popp, and J. I. Cirac, Entanglement versus Correlations in Spin Systems, Phys. Rev. Lett. 92, 027901 (2004).

[35] H. Bombin and M. A. Martin-Delgado, Topological Quantum Distillation, Phys. Rev. Lett. 97, 180501 (2006).

[36] B. M. Terhal, Quantum error correction for quantum memories, Rev. Mod. Phys. 87, 307 (2015).

[37] D. Gottesman, Ph.D. thesis, arXiv:9705052(1997) (1997).

[38] D. Aharonov and M. Ben-Or, Fault-tolerant quantum computation with constant error, SIAM J. Comput. 38, 1207 (1998).

[39] R. Chao and B. W. Reichardt, Quantum Error Correction with Only Two Extra Qubits, Phys. Rev. Lett. 121, 050502 (2018).

[40] C. Chamberland and M. E. Beverland, Flag fault-tolerant error correction with arbitrary distance codes, Quantum 2, 53 (2018).

[41] R. Chao and B. W. Reichardt, Flag fault-tolerant error correction for any stabilizer code, PRX Quantum 1, 010302 (2020).

[42] D. M. Greenberger, M. A. Horne, and A. Zeilinger, in Bell's Theorem, Quantum Theory and Conceptions of the Universe, edited by M. Kafatos (Springer Netherlands, Dordrecht, 1989), p. 69.

[43] D. P. DiVincenzo and P. W. Shor, Fault-Tolerant Error Correction with Efficient Quantum Codes, Phys. Rev. Lett. 77, 3260 (1996).
[44] D. P. DiVincenzo and P. Aliferis, Effective FaultTolerant Quantum Computation with Slow Measurements, Phys. Rev. Lett. 98, 020501 (2007).

[45] A. Bermudez, X. Xu, M. Gutiérrez, S. C. Benjamin, and M. Müller, Fault-tolerant protection of near-term trappedion topological qubits under realistic noise sources, Phys. Rev. A 100, 062307 (2019).

[46] J. Eisert and D. Gross, in Lectures on Quantum Information (John Wiley \& Sons, Ltd, Weinheim, 2007), Chap. 13, p. 237.

[47] R. F. Werner, Quantum states with einstein-podolskyRosen correlations admitting a hidden-variable model, Phys. Rev. A 40, 4277 (1989).

[48] F. Shahandeh, J. Sperling, and W. Vogel, Structural Quantification of Entanglement, Phys. Rev. Lett. 113, 260502 (2014).

[49] Some authors (see, e.g., Refs. [105,106]) define the genuine multipartite entangled states as those not lying within the closed convex hull of all bipartitions. This means that a mixture of two biseparable states with respect to two different bipartitions is still considered biseparable even if it is not separable within any of the two bipartitions. We note, however, that the entanglement of a quantum state with respect to different bipartitions cannot be changed via LOCC and thus, a mixed state of the mentioned form has nonlocal properties beyond biseparability.

[50] I. Bengtsson and K. Zyczkowski, Geometry of Quantum States: An Introduction to Quantum Entanglement (Cambridge University Press, New York, 2006).

[51] M. Horodecki, P. Horodecki, and R. Horodecki, Separability of n-particle mixed states: Necessary and sufficient conditions in terms of linear maps, Phys. Lett. A 283, 1 (2001).

[52] J. Sperling and W. Vogel, Necessary and sufficient conditions for bipartite entanglement, Phys. Rev. A 79, 022318 (2009), 0805.1318

[53] J. Eisert, P. Hyllus, O. Gühne, and M. Curty, Complete hierarchies of efficient approximations to problems in entanglement theory, Phys. Rev. A 70, 062317 (2004).

[54] A. C. Doherty, P. A. Parrilo, and F. M. Spedalieri, Detecting multipartite entanglement, Phys. Rev. A 71, 032333 (2005).

[55] O. Gühne, M. Reimpell, and R. F. Werner, Estimating Entanglement Measures in Experiments, Phys. Rev. Lett. 98, 110502 (2007).

[56] M. Popp, F. Verstraete, M. A. Martín-Delgado, and J. I. Cirac, Localizable entanglement, Phys. Rev. A 71, 042306 (2005).

[57] M. Van den Nest, A. Miyake, W. Dür, and H. J. Briegel, Universal Resources for Measurement-Based Quantum Computation, Phys. Rev. Lett. 97, 150504 (2006).

[58] H.-J. Briegel, W. Dür, J. I. Cirac, and P. Zoller, Quantum Repeaters: The Role of Imperfect Local Operations in Quantum Communication, Phys. Rev. Lett. 81, 5932 (1998).

[59] F. Shahandeh, M. Ringbauer, M. Proietti, F. Costa, A. P. Lund, F. Graffitti, P. Barrow, A. Pickston, D. Kundys, T. C. Ralph, and A. Fedrizzi, Assisted macroscopic quantumness, arXiv:1711.10498 [quant-ph] (2019). 
[60] M. Mičuda, D. Koutný, M. Miková, I. Straka, M. Ježek, and L. Mišta, Experimental demonstration of a fully inseparable quantum state with nonlocalizable entanglement, Sci. Rep. 7, 45045 (2017).

[61] M. Zwerger, W. Dür, J.-D. Bancal, and P. Sekatski, Device-Independent Detection of Genuine Multipartite Entanglement for all Pure States, Phys. Rev. Lett. 122, 060502 (2019).

[62] D. Amaro, M. Müller, and A. K. Pal, Estimating localizable entanglement from witnesses, New J. Phys. 20, 063017 (2018).

[63] D. Amaro, M. Müller, and A. K. Pal, Scalable characterization of localizable entanglement in noisy topological quantum codes, New J. Phys. 22, 053038 (2020).

[64] J. I. Cirac and P. Zoller, Quantum Computations with Cold Trapped Ions, Phys. Rev. Lett. 74, 4091 (1995).

[65] F. Schmidt-Kaler, H. Häffner, M. Riebe, S. Gulde, G. P. T. Lancaster, T. Deuschle, C. Becher, C. F. Roos, J. Eschner, and R. Blatt, Realization of the Cirac-Zoller controlledNOT quantum gate, Nature 422, 408 (2003).

[66] C. D. Bruzewicz, J. Chiaverini, R. McConnell, and J. M. Sage, Trapped-ion quantum computing: Progress and challenges, Appl. Phys. Rev. 6, 021314 (2019).

[67] H. Häffner, C. Roos, and R. Blatt, Quantum computing with trapped ions, Phys. Rep. 469, 155 (2008).

[68] J. Chiaverini, D. Leibfried, T. Schaetz, M. D. Barrett, R. B. Blakestad, J. Britton, W. M. Itano, J. D. Jost, E. Knill, C. Langer, R. Ozeri, and D. J. Wineland, Realization of quantum error correction, Nature 432, 602 (2004).

[69] P. Schindler, J. T. Barreiro, T. Monz, V. Nebendahl, D. Nigg, M. Chwalla, M. Hennrich, and R. Blatt, Experimental repetitive quantum error correction, Science 332, 1059 (2011)

[70] R. Stricker, D. Vodola, A. Erhard, L. Postler, M. Meth, M. Ringbauer, P. Schindler, T. Monz, M. Müller, and R. Blatt, Experimental deterministic correction of qubit loss, Nature 585, 207 (2020).

[71] A. Erhard, H. P. Nautrup, M. Meth, L. Postler, R. Stricker, M. Ringbauer, P. Schindler, H. J. Briegel, R. Blatt, N. Friis, and T. Monz, Entangling logical qubits with lattice surgery, arXiv:2006.03071 [quant-ph] (2020).

[72] M. Gutiérrez, M. Müller, and A. Bermúdez, Transversality and lattice surgery: Exploring realistic routes toward coupled logical qubits with trapped-ion quantum processors, Phys. Rev. A 99, 022330 (2019).

[73] P. Murali, D. M. Debroy, K. R. Brown, and M. Martonosi, Architecting noisy intermediate-scale trapped ion quantum computers, arXiv:2004.04706 [quant-ph] (2020).

[74] N. C. Brown and K. R. Brown, Leakage mitigation for quantum error correction using a mixed qubit scheme, Phys. Rev. A 100, 032325 (2019).

[75] Y. Wang, M. Um, J. Zhang, S. An, M. Lyu, J.-N. Zhang, L.-M. Duan, D. Yum, and K. Kim, Single-qubit quantum memory exceeding ten-minute coherence time, Nat. Photonics 11, 646 (2017).

[76] T. P. Harty, D. T. C. Allcock, C. J. Ballance, L. Guidoni, H. A. Janacek, N. M. Linke, D. N. Stacey, and D. M. Lucas, High-Fidelity Preparation, Gates, Memory, and Readout of a Trapped-Ion Quantum bit, Phys. Rev. Lett. 113, 220501 (2014).
[77] C. J. Ballance, T. P. Harty, N. M. Linke, M. A. Sepiol, and D. M. Lucas, High-Fidelity Quantum Logic Gates Using Trapped-Ion Hyperfine Qubits, Phys. Rev. Lett. 117, 060504 (2016).

[78] H. Kaufmann, T. Ruster, C. T. Schmiegelow, M. A. Luda, V. Kaushal, J. Schulz, D. von Lindenfels, F. Schmidt-Kaler, and U. G. Poschinger, Scalable Creation of Long-Lived Multipartite Entanglement, Phys. Rev. Lett. 119, 150503 (2017)

[79] V. Kaushal, B. Lekitsch, A. Stahl, J. Hilder, D. Pijn, C. Schmiegelow, A. Bermudez, M. Müller, F. SchmidtKaler, and U. Poschinger, Shuttling-based trapped-ion quantum information processing, AVS Quantum Sci. 2, 014101 (2020).

[80] C. J. Ballance, V. M. Schäfer, J. P. Home, D. J. Szwer, S. C. Webster, D. T. C. Allcock, N. M. Linke, T. P. Harty, D. P. L. Aude Craik, D. N. Stacey, A. M. Steane, and D. M. Lucas, Hybrid quantum logic and a test of bell's inequality using two different atomic isotopes, Nature 528, 384 (2015).

[81] K. Mølmer and A. Sørensen, Multiparticle Entanglement of Hot Trapped Ions, Phys. Rev. Lett. 82, 1835 (1999).

[82] A. Sørensen and K. Mølmer, Entanglement and quantum computation with ions in thermal motion, Phys. Rev. A 62, 022311 (2000).

[83] C. A. Sackett, D. Kielpinski, B. E. King, C. Langer, V. Meyer, C. J. Myatt, M. Rowe, Q. A. Turchette, W. M. Itano, D. J. Wineland, and C. Monroe, Experimental entanglement of four particles, Nature 404, 256 (2000).

[84] D. Leibfried, B. DeMarco, V. Meyer, D. Lucas, M. Barrett, J. Britton, W. M. Itano, B. Jelenkovic, C. Langer, T. Rosenband, and D. J. Wineland, Experimental demonstration of a robust, high-fidelity geometric two ion-qubit phase gate, Nature 422, 412 (2003).

[85] U. G. Poschinger, G. Huber, F. Ziesel, M. Deiß, M. Hettrich, S. A. Schulz, K. Singer, G. Poulsen, M. Drewsen, R. J. Hendricks, and F. Schmidt-Kaler, Coherent manipulation of a ${ }^{40} \mathrm{Ca}^{+}$spin qubit in a micro ion trap, J. Phys. B: At. Mol. Opt. Phys. 42, 154013 (2009).

[86] V. Nebendahl, H. Häffner, and C. F. Roos, Optimal control of entangling operations for trapped-ion quantum computing, Phys. Rev. A 79, 012312 (2009).

[87] P. Schindler et al., A quantum information processor with trapped ions, New J. Phys. 15, 123012 (2013).

[88] T. Ruster, C. T. Schmiegelow, H. Kaufmann, C. Warschburger, F. Schmidt-Kaler, and U. G. Poschinger, A long-lived Zeeman trapped-ion qubit, Appl. Phys. B 122, 254 (2016).

[89] D. Kielpinski, C. Monroe, and D. J. Wineland, Architecture for a large-scale ion-trap quantum computer, Nature 417, 709 (2002).

[90] A. Walther, F. Ziesel, T. Ruster, S. T. Dawkins, K. Ott, M. Hettrich, K. Singer, F. Schmidt-Kaler, and U. Poschinger, Controlling Fast Transport of Cold Trapped Ions, Phys. Rev. Lett. 109, 080501 (2012).

[91] T. Ruster, C. Warschburger, H. Kaufmann, C. T. Schmiegelow, A. Walther, M. Hettrich, A. Pfister, V. Kaushal, F. Schmidt-Kaler, and U. G. Poschinger, Experimental realization of fast ion separation in segmented paul traps, Phys. Rev. A 90, 033410 (2014). 
[92] A. Streltsov, U. Singh, H. S. Dhar, M. N. Bera, and G. Adesso, Measuring Quantum Coherence with Entanglement, Phys. Rev. Lett. 115, 020403 (2015).

[93] A remark may be in order here. Throughout the paper "inconclusive" means we can neither conclude the separability nor the entanglement of the state. Recall that this can also be the case if the separability bound lies within the confidence region of the estimated witness value.

[94] L. Egan, D. M. Debroy, C. Noel, A. Risinger, D. Zhu, D. Biswas, M. Newman, M. Li, K. R. Brown, M. Cetina, and C. Monroe, Fault-tolerant operation of a quantum error correction code, arXiv:2009.11482 [quant-ph] (2021).

[95] A. Erhard, H. Poulsen Nautrup, M. Meth, L. Postler, R. Stricker, M. Stadler, V. Negnevitsky, M. Ringbauer, P. Schindler, H. J. Briegel, R. Blatt, N. Friis, and T. Monz, Entangling logical qubits with lattice surgery, Nature 589, 220 (2021).

[96] M. Kjaergaard, M. E. Schwartz, J. Braumüller, P. Krantz, J. I.-J. Wang, S. Gustavsson, and W. D. Oliver, Superconducting qubits: Current state of play, Annu. Rev. Condens. Matter Phys. 11, 369 (2020).

[97] A. Browaeys and T. Lahaye, Many-body physics with individually controlled Rydberg atoms, Nat. Phys. 16, 132 (2020).

[98] M. Veldhorst, J. C. C. Hwang, C. H. Yang, A. W. Leenstra, B. de Ronde, J. P. Dehollain, J. T. Muhonen, F. E. Hudson, K. M. Itoh, A. Morello, and A. S. Dzurak, An address- able quantum dot qubit with fault-tolerant control-fidelity, Nat. Nanotechnol. 9, 981 (2014).

[99] C. E. Bradley, J. Randall, M. H. Abobeih, R. C. Berrevoets, M. J. Degen, M. A. Bakker, M. Markham, D. J. Twitchen, and T. H. Taminiau, A Ten-Qubit SolidState Spin Register with Quantum Memory up to one Minute, Phys. Rev. X 9, 031045 (2019).

[100] S. Takeda and A. Furusawa, Toward large-scale faulttolerant universal photonic quantum computing, APL Photonics 4, 060902 (2019).

[101] M. Walter, D. Gross, and J. Eisert, Multi-partite entanglement, arXiv:1612.02437 [quant-ph] (2017).

[102] T. Durt, D. Kaszlikowski, J.-L. Chen, and L. C. Kwek, Security of quantum key distributions with entangled qudits, Phys. Rev. A 69, 032313 (2004).

[103] C. Schaeff, R. Polster, M. Huber, S. Ramelow, and A. Zeilinger, Experimental access to higher-dimensional entangled quantum systems using integrated optics, Optica 2, 523 (2015).

[104] J. Wang et al., Multidimensional quantum entanglement with large-scale integrated optics, Science 360, 285 (2018).

[105] O. Gühne and G. Tóth, Entanglement detection, Phys. Rep. 474, 1 (2009).

[106] M. Huber, F. Mintert, A. Gabriel, and B. C. Hiesmayr, Detection of High-Dimensional Genuine Multipartite Entanglement of Mixed States, Phys. Rev. Lett. 104, 210501 (2010). 